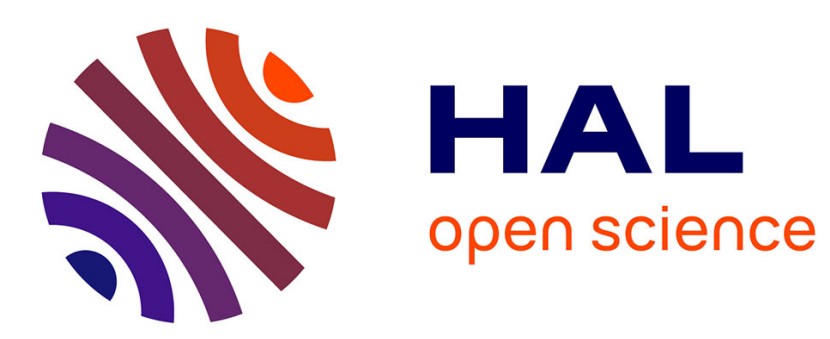

\title{
Impurity bands and quasi-Bloch waves for a one-dimensional model of modulated crystal Laurent Gosse
}

\section{To cite this version:}

Laurent Gosse. Impurity bands and quasi-Bloch waves for a one-dimensional model of modulated crystal. Nonlinear Analysis: Real World Applications, 2008, 9 (3), pp.927-948. 10.1016/j.nonrwa.2007.01.012 . hal-00432205

\section{HAL Id: hal-00432205 https://hal.science/hal-00432205}

Submitted on 14 Nov 2009

HAL is a multi-disciplinary open access archive for the deposit and dissemination of scientific research documents, whether they are published or not. The documents may come from teaching and research institutions in France or abroad, or from public or private research centers.
L'archive ouverte pluridisciplinaire HAL, est destinée au dépôt et à la diffusion de documents scientifiques de niveau recherche, publiés ou non, émanant des établissements d'enseignement et de recherche français ou étrangers, des laboratoires publics ou privés. 


\title{
Impurity bands and quasi-Bloch waves for a one-dimensional model of modulated crystal
}

\author{
Laurent Gosse* \\ IAC-CNR "Mauro Picone" (sezione di Bari) \\ Via Amendola 122/D - 70126 Bari, Italy
}

\begin{abstract}
This paper investigates a simple one-dimensional model of incommensurate "harmonic crystal" in terms of the spectrum of the corresponding Schrödinger equation. Two angles of attack are studied: the first exploits techniques borrowed from the theory of quasi-periodic functions while the second relies on periodicity properties in a higher-dimensional space. It is shown that both approaches lead to essentially the same results; that is, the lower spectrum is splitted between "Cantor-like zones" and "impurity bands" to which correspond critical and extended eigenstates, respectively. These "new bands" seem to emerge inside the band gaps of the unperturbed problem when certain conditions are met and display a parabolic nature. Numerical tests are extensively performed on both steady and time-dependent problems.
\end{abstract}

Key words: Quasi-periodic potential, impurity band, quasi-Bloch wave, quantum chaos, spectral algorithm, deformed crystal, Charge-Density Wave (CDW). 1991 MSC: 81Q05, 81Q20

\section{Introduction}

This article is a step further in the numerical study of electronic motion in a one-dimensional model of "harmonic crystal", that is to say, a modeling in solid-state physics going beyond the rigid periodic lattice picture. Indeed, it consists in assuming that, within the BornOppenheimer approximation (the motion of nucleons is much slower than the electron's ones and thus decouples adiabatically), ionic cores are linked together by springs and vibrate like classical coupled oscillators; consult the book [1] and the recent paper [14] to which we are about to borrow notation.

In [14], the following rescaled problem describing the motion of a free electron inside a 1-D lattice of atoms which display single-mode vibrations has been investigated within the

\footnotetext{
* Corresponding Author

Email address: 1.gosse@ba.iac.cnr.it (Laurent Gosse).
} 
WKB semi-classical framework $[5,19]$ for the following Schrödinger equation,

$$
i \lambda \partial_{t} \psi+\frac{\lambda^{2}}{2} \partial_{x x} \psi=\cos \left(\frac{x}{\lambda}-\varepsilon \sin \left(\frac{k x}{\lambda}-\Omega(k) t\right)\right) \psi, \quad \varepsilon \ll 1, \lambda \ll 1
$$

and under the fundamental assumption that $k$ and $\Omega(k)=|\sin (k \pi)|$ are rational and commensurate. This is perhaps the simplest non-trivial model available which describes the effects of phonons onto electronic conduction and is sometimes referred to as the "modulated crystal" $[1,9,25,26,32]$. Since the potential in (1) isn't of the form $\cos \left(x-\frac{1}{2} a t^{2}\right)$, the unitary transform propsed in [35] and leading to Bloch oscillations doesn't apply here (see also [33] for an AC-type modulation). A particular feature pointed out in [39] and called lattice tracking which refers to the dragging effect of heavy nucleons onto the much lighter electron was easily observed in the numerical results.

The commensurability hypothesis is of critical importance in this global picture in order to keep on applying the classic Bloch decomposition for (1); we assume the reader familiar with this theory and refer only to $[1,5,15,19]$ for details. The first step in this line of thinking is the derivation of "energy bands", which are dispersion relations for electrons around certain levels of excitation. Solids are classified in metals, semi-conductors and insulators depending if the Fermi energy level lies inside a partially filled conduction band or somewhere inside a gap being of moderate or big size, respectively. Generally, semi-classical approximation restricts itself to considering only the propagation of charge carriers in valence and conduction bands: lower ones correspond to inner shells electrons strongly tied to the atomic cores and higher ones are usually empty except in special cases of intense excitation. It has been observed that the use of modern shock-capturing numerical techniques allow for quite satisfying computations of wave packets in increasingly complex environments including possibly self-interactions: see [14-18].
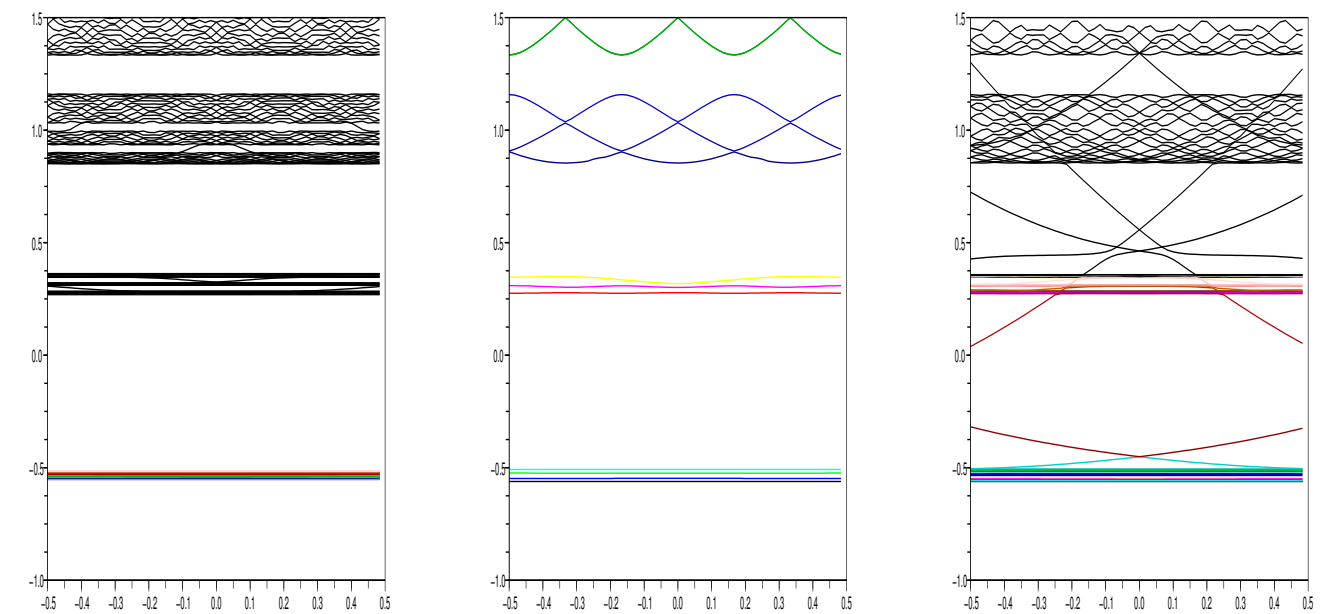

Fig. 1. Band structures for $\varepsilon=0.15$ with commensurate (middle) and incommensurate models: $k=\frac{\sqrt{5}-1}{2}$ (left), $k=\frac{2}{\sqrt{5}-1}$ (right). Abscissas and ordinates correspond to the "quasi-momentum" $\kappa$ and the energies $E$ in (6) respectively.

However, a simple linearization around the Mathieu (or Hill) potential in (1) reveals that in case $k$ and $\Omega(k)$ become incommensurate, one has to deal with a quasi-periodic perturbation $^{1}$ inside the Schrödinger equation. It is commonly believed that as long as the

1 A precise definition of this term is to be given in $\S 2.1$ of this text. 
strength of the perturbation remains small (how small ?), its effect onto the energy bands is negligible and corresponding Bloch states vibrate according to the same wavenumber $k$, see e.g. [39] §IV. One could argue that this would be at best a perturbative KAM-type ${ }^{2}$ result, expressing the fact that the Hamiltonian structure in phase space remains mostly unaltered. We intend to show here that this picture is overmuch simplified. New phenomena can and do occur according to the modulated potential in (1): to convince at once the reader of this fact, we present in Fig. 1 the band structure of (1) for $k=\frac{5}{3}, k=\frac{2}{\sqrt{5}-1}$ (the so-called golden mean) and its inverse inside the Brillouin zone of the classical Mathieu equation, i.e. $\left[-\frac{1}{2}, \frac{1}{2}\right]$. Comparing with Fig. 1 in [15], hard would it be to see anything they might share!

Looking more carefully at the picture on the right corresponding to the golden mean, one may observe an amazing phenomenon: inside the intervals occupied by the energy bands of the unperturbed Mathieu equation, messy states appear whereas it is exactly inside the "exband gaps" that seemingly new bands show up. How could this be heuristically explained ? First, let us stress the fact that, in case $k=\frac{5}{3}$, the perturbed potential remains periodic in the $x$ variable with $t$ fixed, even if the period has become quite big. Consequently, the real Brillouin zone (like the ones considered previously in [14]) is tiny. Hence looking at this scale can give the feeling that these "new bands" look like constant states related to an isolated impurity (this has a lot to do with the "hydrogenic donor" approximation, see [1,37] and Fig. 9 in [16]). Obviously, when $k$ has become totally irrational, the real Brillouin zone is reduced to a point. Further, one sees on the right picture of Fig. 1 that original (unperturbed) bands have completely collapsed: this is a consequence of a phenomenon called band nesting and beautifully illustrated in [21]. Loosely speaking, it refers to a mechanism which creates smaller and smaller "mini-gaps" inside a given band thus splitting it into a Cantor-like structure, see again $[26,23]$.

In $\S 2.1$, we shall review briefly the rigorous mathematical results on eigenfunctions for quasi-periodic 1-D problems which followed the seminal article by Dinaburg and Sinai, [7]. They all rely on difficult KAM arguments and are mostly concerned with bounded solutions of the quasi-periodic Hill's equation $y^{\prime \prime}+(E-\epsilon q(x))=0$, for small $\epsilon$ or high $E$, see $[30,22,11,40,8]$. However, our situation here is slightly different as we are dealing with a periodic Hill equation with a (possibly small) incommensurate perturbation added: our main theoretical references should be therefore [3,12], even if it doesn't exactly fit in $(1) . \S 2.2$ is devoted to presenting a spectral algorithm to compute eigenvalues and eigenfunctions in the quasi-Bloch wave form. $\S 3$ presents some results for a simple quasi-periodic potential of the Mathieu type: the main emphasis is given to the appearance of "new bands" which look like being $k$-periodic, see Figs. 2 and 3 . In $\S 4$, we study eigenvalues and eigenfunctions for the equation (1). Again, when $k$ is incommensurate, we observe $k$-periodic "new bands" which are seemingly parabolic, see especially Fig. 8. To these "new parabolic bands" correspond eigenstates which are extended, in sharp contrast to the critical states associated to the lower levels of Cantor-type nature as explained in $\S 4.4$. We shall even see that these critical states are localized on the iso-values of the potential. In $\S 5$, we study briefly what happens for different values of $k$.

Let us close this introduction emphasizing that defining a linearized potential in (1) like

$$
V(t, x) \stackrel{\text { def }}{=} \cos (x)+\varepsilon \sin (x) \sin (k x-\Omega(k) t), \quad 0 \leq \varepsilon \ll 1,
$$

there are still periodicity properties that can be exploited:

$$
V(t+2 \pi / \Omega(k), x)=V(t, x), \quad V(t+2 k \pi / \Omega(k), x+2 \pi)=V(t, x) .
$$

This fact, already noticed in [26], opens the way to perform a "quasi-Bloch decomposition" in 2-D for problems of the flavour of (1) for arbitrary values of $k$. The price to pay is of course a much higher computational cost as two indices will show up in the corresponding

2 for Kolmogorov, Arnold and Moser. 
Fourier series; but it also turns out that steady quasi-periodic problems ask for the same effort (see [40] for an example and Appendix A for the corresponding illustration).

Remark 1 In every figure displaying a band structure, like Fig. 1, abscissas will always correspond to the Bloch "quasi-momentum" denoted hereafter and in (6) by $\kappa$. Ordinates stand for the associated energies $E$ respectively.

\section{Theoretical framework for "quasi-Bloch" decompositions}

There are (at least) two ways to attack the eigen-functions problem given by a potential like (2): one follows techniques borrowed from existing papers on quasi-periodic equations, the other tries to take advantage of the space/time periodic structure (3) that still remains. We shall see in the sequel that both lead essentially (and fortunately) to the same algorithm in the two-dimensional case, but let us first recall some basic "quasi-periodic techniques".

\subsection{Quasi-periodic potentials: a short overview of known results}

Following [36], let us begin with:

Definition 1 Let $q(x)$ be a smooth function $\mathbb{R} \rightarrow \mathbb{R} ; q$ is said to be quasi-periodic if there exist $d \in \mathbb{N}$ constants $\vec{\omega}=\left(\omega_{1}, \ldots, \omega_{d}\right) \in \mathbb{R}^{d}$ and a continuous function $Q: \mathbb{R}^{d} \rightarrow \mathbb{R} 2 \pi-$ periodic in each of its arguments such that:

$$
\forall x \in \mathbb{R}, \quad q(x)=Q(\vec{\omega} x) \stackrel{\text { def }}{=} Q\left(\omega_{1} x, \ldots, \omega_{d} x\right)
$$

The $d$ constants $\vec{\omega}$ are called the basic frequencies of $q$ and $Q$ is the (non unique) lift of $q$.

When dealing with quasi-periodic functions, we shall always use capital letters for the corresponding lifts. It is generally assumed that the components of $\vec{\omega}$ are rationally independent otherwise the function $q$ is simply periodic and $Q$ might become ambiguous. Notice that this definition is compatible with the one we gave in [15]; it is a bit more general though.

It may sometimes be interesting to work with quasi-periodic functions endowed with an "initial phase" $\phi \in \mathbb{R}^{d}$, for which $q_{\phi}(x)=Q(\vec{\omega} x+\phi)$. Roughly speaking, one can figure out what quasi-periodic functions are by thinking about functions of a single real variable admitting a multi-dimensional Fourier series: indeed, we write

$$
q(x)=\sum_{\vec{k} \in \mathbb{Z}^{d}} \hat{Q}_{\vec{k}} \exp (i<\vec{k}, \vec{\omega} x>), \quad \hat{Q}_{\vec{k}}=\frac{1}{(2 \pi)^{d}} \int_{\mathbb{T}^{d}} Q(\theta) \exp (-i<\vec{k}, \theta>) \cdot d \theta
$$

The notation $<., .>$ stands for the scalar product in $\mathbb{R}^{d}$ and the $d$-dimensional torus $\mathbb{T}^{d}$, for $[0,2 \pi]^{d}$. It is generally assumed that the Fourier series (4) converges uniformly ${ }^{3}$. In this context, it makes sense to wonder if the elegant Floquet-Bloch theory for the onedimensional periodic ${ }^{4}$ Schrödinger equation

$$
y^{\prime \prime}+(E-\epsilon q(x))=0, \quad y(x) \text { polynomially bounded }
$$

3 This trongly depends on the smoothness of $Q$.

4 This is the case where $d=1$. 
extends in some sense to quasi-periodic potentials $q$. As soon as $q$ is bounded, general spectral theory ensures that the corresponding operator is essentially self-adjoint on $\mathbb{R}$; we shall denote $\mathcal{H}$ its self-adjoint extension to $L^{2}(\mathbb{R})$.

Proposition 1 ([36]) Let $q_{\phi}(x)=Q(\vec{\omega} x+\phi)$ where $Q$ is continuous $\mathbb{T}^{d} \rightarrow \mathbb{R}$ and $\vec{\omega}$ is rationally independent. Then the spectrum of the corresponding Schrödinger operator $\mathcal{H}$ on $L^{2}(\mathbb{R})$ doesn't depend on $\phi$. Moreover, it doesn't have any isolated eigenvalues.

The study of quantum particles submitted an incommensurate potential started with [34] and electrons moving inside a magnetic field. In a nutshell, the theory for quasi-periodic (5) states that both eigenfunctions and eigenvalues $E$ strongly depend on $\epsilon$. For large $\epsilon$, eigenfunctions are known to decay exponentially, this phenomenon is referred to as Anderson localization, see e.g. [11]. But for small $\epsilon$ and/or big E, there exist quasi-Bloch waves, that is to say eigenfunctions of the form (according to the former notation),

$$
y(x)=\exp (i \kappa(E) x) z(x), \quad z(x)=Z(\vec{\omega} x),
$$

the lift $Z$ being $2 \pi$-periodic in each of its arguments. KAM techniques are used in the proofs, which means that small divisors are to be controlled during the process. To do so, it is necessary to assume that $\vec{\omega}$ is Diophantine, i.e. there exist constants $C_{0}, \tau$ for which,

$$
\forall \vec{\nu} \neq \overrightarrow{0} \in \mathbb{Z}^{d}, \quad|<\vec{\omega}, \vec{\nu}>| \geq C_{0}|\vec{\nu}|^{-\tau}, \quad|\kappa(E)+<\vec{\omega}, \vec{\nu}>| \geq C_{0}|\vec{\nu}|^{-\tau}
$$

Quasi-Bloch waves for (5) with $\left.\kappa(E)=\frac{1}{2}<\vec{\omega}, \vec{t}\right\rangle$, with $\vec{t} \notin \mathbb{Z}^{d}$ and $|\langle\vec{\omega}, \vec{t}\rangle| \geq C_{0}|\vec{t}|^{-\tau}$ have been found in $[7,22,30,8]$ along with the presence of gaps in the spectrum. Actually, to any quasi-periodic potential $q$ and any eigenvalue $E^{*}$ can be associated a rotation number

$$
\beta\left(\epsilon, E^{*}\right)=(2 \pi)^{-d} \lim _{x \rightarrow \infty} \frac{1}{x} \arg \left(y^{\prime}(x)+i y(x)\right)
$$

where $y$ solves (5) for the particular eigenvalue $E^{*}$; this number is independent of the selected solution $y$. It realizes a continuous function $\mathbb{R}^{2} \rightarrow \mathbb{R}$. Moreover, it is a nondecreasing function of $E^{*}$ for $\epsilon$ fixed. The Gap Labelling theorem expresses the fact that $\beta(\epsilon, E)$ is constant inside any connected component of the resolvent set:

Theorem 1 ([22]) Let $q$ be quasi-periodic with basic frequencies $\vec{\omega}$ and $I$, an open interval inside a spectral gap. There exist integers $\vec{k} \in \mathbb{Z}^{d}$ such that:

$$
\forall E \in I, \quad \beta(\epsilon, E)=\frac{1}{2}<\vec{k}, \vec{\omega}>
$$

The converse is also true: intervals of constancy for $\beta$ are exactly the spectral gaps of $\mathcal{H}$.

Definition 2 ([3]) Let $\vec{k} \in \mathbb{Z}^{d}$; the resonance tongue of (5) associated to $\vec{k}$ is the set:

$$
\mathcal{R}(\vec{k})=\left\{(\epsilon, E) \in \mathbb{R}^{2} \text { such that } \beta(\epsilon, E)=\frac{1}{2}<\vec{k}, \vec{\omega}>\right\} .
$$

This notion is interesting because it helps in validating numerical results indicating where spectral gaps can be for small $\varepsilon$, see [4,3]; we shall use it in Appendix A. Recently, an extension to the slightly more involved situation

$$
y^{\prime \prime}+(E-p(x)-\epsilon q(x))=0, \quad x \in \mathbb{R},
$$

where $p$ is periodic has been treated in [12] (see also [3]); it led to the same kind of results, but for completeness we state the theorem: 
Theorem 2 ([12]) Let $p: \mathbb{R} \rightarrow \mathbb{R}$ be real analytic and periodic with frequency $\omega_{0} \in \mathbb{R}$ and such that the fundamental solutions of $y^{\prime \prime}+p(x)=0$ are quasi-periodic with a proper frequency $\Omega_{0} \in \mathbb{R}$. Let furthermore $q: \mathbb{R} \rightarrow \mathbb{R}$ be real analytic and quasi-periodic with basic frequencies $\vec{\omega} \in \mathbb{R}^{d}$. Define $\tilde{\omega}:=\left(\vec{\omega}, \omega_{0}, \Omega_{0}\right) \in \mathbb{R}^{d+2}$ and assume $\tilde{\omega}$ is rationally independent with, moreover

$$
\forall \vec{\nu} \neq \overrightarrow{0} \in \mathbb{Z}^{d+2}, \quad|<\tilde{\omega}, \vec{\nu}>| \geq C_{0}|\vec{\nu}|^{-\tau},
$$

for two fixed positive constants $C_{0}$ and $\tau>d+1$ (Diophantine conditions). Then there exist $\epsilon_{0}>0$ small and a Cantor set $\mathcal{E} \subset\left[-\epsilon_{0}, \epsilon_{0}\right]$ such that, for all $\epsilon \in \mathcal{E}$, the equation $y^{\prime \prime}+p(x)+\epsilon q(x)=0$ admits a quasi-periodic solution of the form:

$$
y(x)=\exp \left(i \Omega_{\epsilon} x\right) \sum_{\vec{m}, n \in \mathbb{Z}^{d+1}} \tilde{y}_{\vec{m}, n}(\epsilon) \exp \left(i\left(<\vec{m}, \vec{\omega}>+n \omega_{0}\right) x\right),
$$

and the above sum is absolutely and uniformly convergent for all $x \in \mathbb{R}$ and $\epsilon \in \mathcal{E}$.

Clearly (9) is a quasi-Bloch wave in the aforementioned sense. In the sequel, we shall mainly use Theorem 2 with $d=1$. Let us finally stress that either (6) (and thus (9)) do agree with the ansatz suggested by experimentalists, see the conclusion of [38] and [42].

Remark 2 Theorem 2 can be recast into Eliasson's theory as we briefly explain now: any periodic Hill's equation is endowed with Floquet reducibility, meaning that a periodic change of variables takes it to constant coefficients. The existence of Bloch waves depends on the form of this constant coefficient Floquet matrix. Now, considering the quasi-periodic problem (8) and performing periodic reducibility, one obtains a small $\epsilon$-quasi-periodic perturbation of a matrix with constant coefficients to which Eliasson's results can be applied.

\subsection{An algorithm adapted to quasi-periodic potentials}

The idea is to extend the spectral algorithm used in $[15,16]$ to compute eigenvalues and Bloch waves in the periodic case to the more involved case of potentials $q$ of the type (4). Indeed, assuming there exist quasi-Bloch waves solving the eigenvalue problem for $\mathcal{H}$, we can project the differential equation onto the Fourier base corresponding to $q$. Taking into account for the quasi-periodic modulation $z(x)$, we see that $(6)$ rewrites

$$
\Psi(x)=\sum_{\vec{k} \in \mathbb{Z}^{d}} \hat{Z}_{\vec{k}} \exp (i(<\vec{k}, \vec{\omega}>+\kappa(E)) x),
$$

where the coefficients of the lift $\hat{Z}_{\vec{k}}$ are computed as in (4):

$$
\forall \vec{k} \in \mathbb{Z}^{d}, \quad \hat{Z}_{\vec{k}}=\frac{1}{(2 \pi)^{d}} \int_{\mathbb{T}^{d}} Z(\theta) \exp (-i<\vec{k}, \theta>) \cdot d \theta
$$

At this level, we follow [15], $\S 2.2$ and compute for $\vec{k}, \vec{k}^{\prime} \in \mathbb{Z}^{d}$ :

$$
\int_{\mathbb{T}} \partial_{x x}\{\exp (i(<\vec{k}, \vec{\omega}>+\kappa) x)\} \exp \left(-i\left(<\vec{k}^{\prime}, \vec{\omega}>+\kappa\right) x\right) \cdot d x=-(<\vec{k}, \vec{\omega}>+\kappa)^{2}
$$

where we used the $L^{2}$-orthogonality of the base, and using (4)

$$
\int_{\mathbb{T}}\left(\sum_{\vec{\ell} \in \mathbb{Z}^{d}} \hat{Q}_{\vec{\ell}} \exp (i<\vec{\ell}, \vec{\omega} x>)\right) \exp \left(i<\vec{k}-\vec{k}^{\prime}, \vec{\omega} x>\right) \cdot d x=\hat{Q}_{\vec{k}-\vec{k}^{\prime}}
$$


The eigenvalue problem for $\mathcal{H}$ thus rewrites:

$$
(<\vec{k}, \vec{\omega}>+\kappa)^{2} \hat{Z}_{\vec{k}}+\sum_{\vec{k}^{\prime} \in \mathbb{Z}^{d}} \hat{Q}_{\vec{k}-\vec{k}^{\prime}} \hat{Z}_{\vec{k}^{\prime}}=E(\kappa) \hat{Z}_{\vec{k}}
$$

As long as $\vec{k}, \vec{k}^{\prime}$ belong to a finite set, (11) leads to an eigenvalue problem for a (very big!) structured matrix when the eigenvectors $\hat{Z}_{\vec{k}}$ are arranged in a convenient way. Truncations and error estimates can be computed following the method presented in Chap. 3, $§ 11$ of the book [41], relying on the existence results of [3,12]. Concerning the convergence of the algorithm (11) as $|\vec{k}|,\left|\vec{k}^{\prime}\right| \rightarrow+\infty$, we recall from $[28,46]$ the following definition:

Definition 3 Given any matrix $A=\left(a_{i, j}\right)_{i, j}$ with complex entries, its determinant is said to be of Hill's type if the following condition holds:

$$
\sum_{i, j}\left|a_{i, j}-\delta_{i, j}\right|<+\infty, \quad \delta_{i, j} \text { the Kronecker symbol. }
$$

Dividing each line of (11) by its diagonal term is the first step to study whether the resulting matrix is of Hill's type, for instance when $Q$ is analytic, i.e. $\left|\hat{Q}_{\vec{k}}\right| \leq C_{Q} \exp (-\xi|\vec{k}|)$ or when $Q$ is a finite sum of Fourier modes. Indeed, it comes

$$
\hat{Z}_{\vec{k}}+\sum_{\vec{k}^{\prime} \in \mathbb{Z}^{d}} \frac{\hat{Q}_{\vec{k}-\vec{k}^{\prime}}}{\mid\left\langle\vec{k}, \vec{\omega}>+\kappa-\left.E(\kappa)\right|^{2}\right.} \hat{Z}_{\vec{k}^{\prime}}=0
$$

to which we apply the Diophantine condition (7) stating that:

$$
\forall \vec{k} \in \mathbb{Z}^{d}, \quad \frac{1}{|<\vec{k}, \vec{\omega}>+\kappa|} \leq C_{0}|\vec{k}|^{\tau}
$$

And the behavior for large Fourier modes with small divisors is ruled by terms of the type $|\vec{k}|^{2 \tau} \exp \left(-\xi\left|\vec{k}-\vec{k}^{\prime}\right|\right)$; other cases are like $|<\vec{k}, \vec{\omega}>+\kappa|^{-2} \exp \left(-\xi\left|\vec{k}-\vec{k}^{\prime}\right|\right)$. Hence if small divisors are very few, condition (12) can be met and according to the theory in [28], the diagonalization process (11) should converge (for the values of $E(\kappa)$ for which the denominator doesn't vanish).

\section{$3 \quad$ A steady quasi-periodic potential}

\subsection{Quasi-periodic perturbation of the Mathieu potential}

The behavior of eigenvalues for the following time-independent equation has been studied theoretically in $[11,40,45]$, numerically in $[6,21,46]$ and experimentally in [44] (see also [42]):

$$
\frac{1}{2} \partial_{x x} \Psi+(E-\varepsilon q(x)) \Psi=0, \quad q(x)=\cos (x)+\cos (k x) .
$$

From $§ 2.1$, we know that, provided the Diophantine condition (7) holds,

$$
\forall m, n \neq 0 \in \mathbb{Z}^{2}, \quad|m+n k| \geq \frac{C_{0}}{n^{2}},
$$


for $\varepsilon$ small enough and/or $E$ big, "quasi-Bloch waves" $\Psi(x)=\exp (i \kappa x) z(\kappa, x)$ do solve the quasi-periodic eigenvalue problem (13). In this last case, $z(\kappa,$.$) and q$ share the same kind of quasi-periodicity: according to (4) they read,

$$
q(x)=\sum_{n, m} \hat{Q}_{n, m} \exp (i(n+m k) x), \quad z(\kappa, x)=\sum_{n, m} \hat{Z}_{n, m}(\kappa) \exp (i(n+m k) x) .
$$

Of course, the potential is very well compressed in this base, $\hat{Q}_{ \pm 1,0}=\frac{1}{2}, \hat{Q}_{0, \pm 1}=\frac{1}{2}$ and the "quasi-momentum" $\kappa(E) \in \mathbb{R}$ doesn't belong to any Brillouin zone. This clearly allows for the use of the spectral algorithm (11) to compute the admissible values of $E$ and their corresponding "quasi-Bloch waves" $\Psi$; we present numerical results in Appendix A. However, by analogy with the original problem (2), we decided to work with a slightly different equation: namely the corresponding potential reads with $\varepsilon \in[0,1]$,

$$
q(x)=\frac{1}{2}(\cos (x)+\varepsilon \cos (k x)), \quad \hat{Q}_{ \pm 1,0}=\frac{1}{4}, \hat{Q}_{0, \pm 1}=\frac{\varepsilon}{4} .
$$

This is rather a "small quasi-periodic perturbation" of a periodic problem hence we follow the ideas recalled in Prop. 2 and the algorithm proposed by Surace extends easily:

$$
(n+m k+\kappa)^{2} \hat{Z}_{n, m}+\frac{1}{2}\left(\hat{Z}_{n-1, m}+\hat{Z}_{n+1, m}+\varepsilon \hat{Z}_{n, m-1}+\varepsilon \hat{Z}_{n, m+1}\right)=2 E_{\kappa} \hat{Z}_{n, m} .
$$

This special case of (11) loops on $\kappa \in[-1,1]$ (say!) and we chosed to restrict the computation to $n, m \in\{-15,15\}$. The eigenvectors read $\left(\hat{Z}_{-15,-15}, \hat{Z}_{-15,-14}, \ldots \hat{Z}_{-15,15}, \hat{Z}_{-14,-15}, \ldots\right)$. The differential equation has therefore been reduced to the diagonalization of a $31^{2} \times 31^{2}$ pentadiagonal symmetric matrix. We consider first two different cases $k=\frac{5}{3}$ and $k=\frac{2}{\sqrt{5}-1}$ : these two numbers are close to each other, but the second one is Diophantine thus enters completely in the theoretical framework of $[12,40]$ which rigorously ensures the existence of quasi-Bloch solutions.

\subsection{Some numerical results with $\varepsilon=0.02$ and $\varepsilon=0.2$}

The numerical outcome $\kappa \mapsto E_{\kappa}$ is presented in Figs. 2 and 3 for $\varepsilon=0.02$ and $\varepsilon=0.2$ respectively. Several comments are in order: first, for $k=5 / 3$ there's no need $^{5}$ for the algorithm (16) as the period of the problem is $6 \pi$. Hence the corresponding Fourier base should be $\exp ($ inx $x / 3)$, the Brillouin zone, $\kappa \in[-1 / 6,1 / 6]$ and the standard procedure explained in $[15,16]$ can be used up to minor changes: its outcome is actually displayed on the left in Figs. 2 and 3. Nevertheless, we also iterated the quasi-periodic algorithm (16) in order to study both its accuracy and its transition between the commensurate and the incommensurate case. We observe that high in the spectrum, bands are always 1/3-periodic as expected; the lower ones should be also, but it isn't visible. However, something does show up inside the band gaps: a "new band" which doesn't look like being $1 / 3$-periodic at all. It seems to survive the "band clustering" that destroys all the other ones when we put $k=\frac{2}{\sqrt{5}-1}$ (on the right in Figs. 2 and 3 ).

For $\varepsilon=0.2$, Fig. 3 reveals the same type of pattern. That is, usual Bloch bands are easily noticeable on the left, and they are 1/3-periodic. There is an agreement between the left and middle figure, except for similar "new bands 6 ". Usual bands collapse when choosing $k=\frac{2}{\sqrt{5}-1}$ because of the band nesting phenomenon illustrated in [21] and become an intricate set that a computer cannot calculate properly. However, the new bands looking

5 Using it even seems to decrease a lot the accuracy of the numerical outcome.

6 which are spurious for $k=5 / 3$, we explain why in $\S 3.3$. 

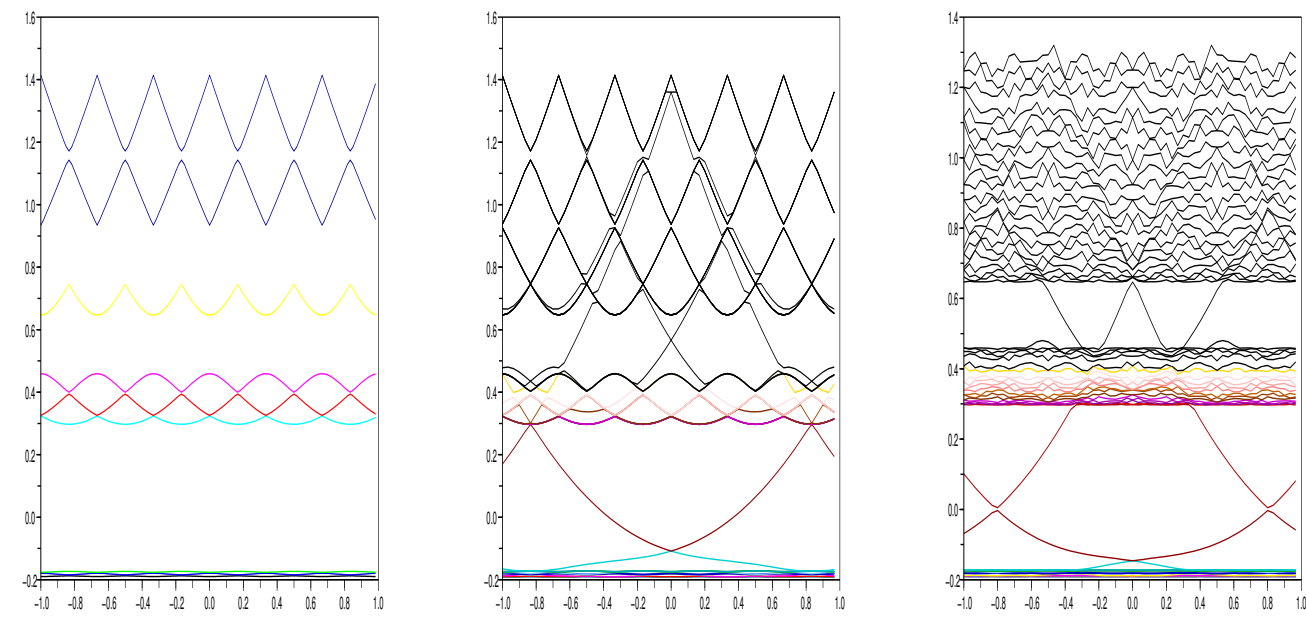

Fig. 2. Band structure with $\varepsilon=0.02$ and $k=5 / 3$ (left and middle), $k=\frac{2}{\sqrt{5}-1}$ (right). The figure on the left is obtained with the optimal Fourier base $\exp (\operatorname{inx} / 3)$.
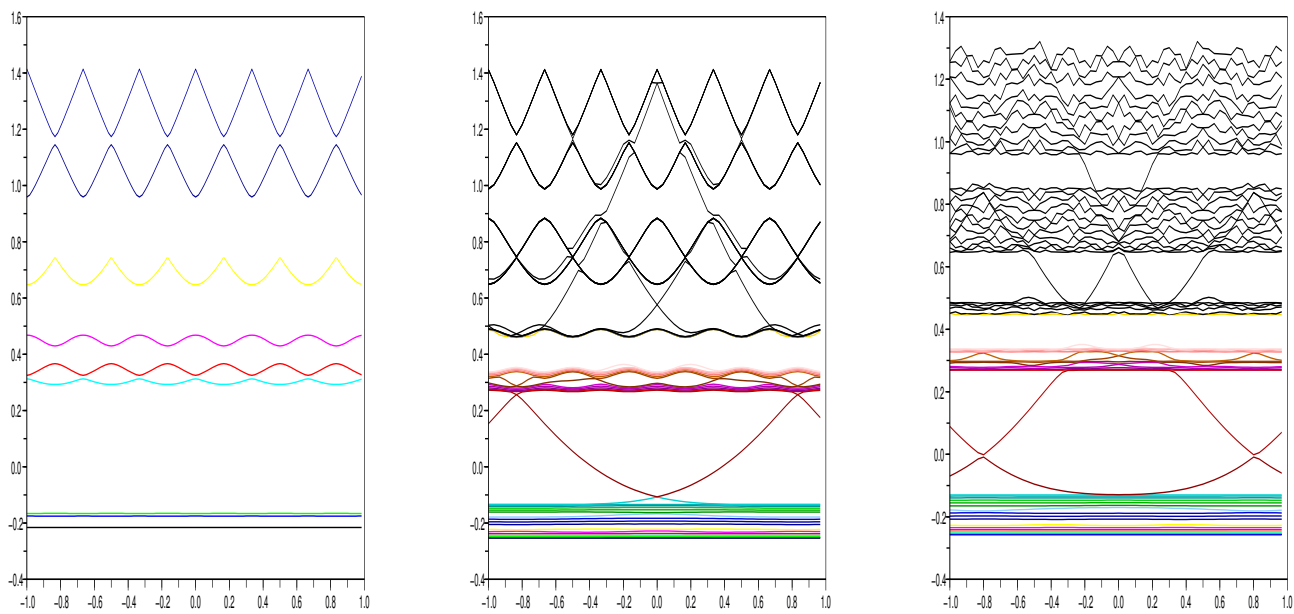

Fig. 3. Band structure with $\varepsilon=0.2$ and $k=5 / 3$ (left and middle), $k=\frac{2}{\sqrt{5}-1}$ (right). The figure on the left is obtained with the optimal Fourier base $\exp (i n x / 3)$.

like being $k$-periodic (roughly repeating themselves from $\kappa=-0.8$ to $\kappa=0.8$ ) survive the process thus remain the only ones on the messy right figure.

\subsection{Impurity bands?}

Concerning the presence of the "new bands" in case $k=5 / 3$ : we know from Floquet-Bloch theory [19] that since the problem is $6 \pi$-periodic, they shouldn't appear and this is what we get when using the algorithm based on $\exp (\operatorname{inx} / 3)$. Especially the problem is quite 
degenerate as many eigenvalues look like being very close to each other: this shows that the choice of the Fourier base by means of which one expresses both $V(x)$ and $\Psi(x)$ is of paramount importance in terms of numerical accuracy. For the same type of reason, putting $\varepsilon=0$ inside (16) isn't likely to lead to satisfying numerical results as the selected Fourier base may not be the optimal one; hence the performances of the (quasi-periodic) algorithm on a periodic problem are quite low. Let us stress that in both cases ( $k$ rational and $\varepsilon=0)$, the algorithm (16) is built by projecting the differential equation (13) on the set of functions $\exp (i(n+m k) x) ; n, m$ in a finite subset of $\mathbb{Z}^{2}$. However, when $\varepsilon=0$, the $m$ index is useless; and when $k=5 / 3$, we notice that for e.g. $n=3, m=0$ and $n=0, m=5, L^{2}$-orthogonality is lost, along with the derivation of (11). This last remark suggests that (16) should work correctly as long as orthogonality is kept within the selected finite set of indices $(n, m)$.
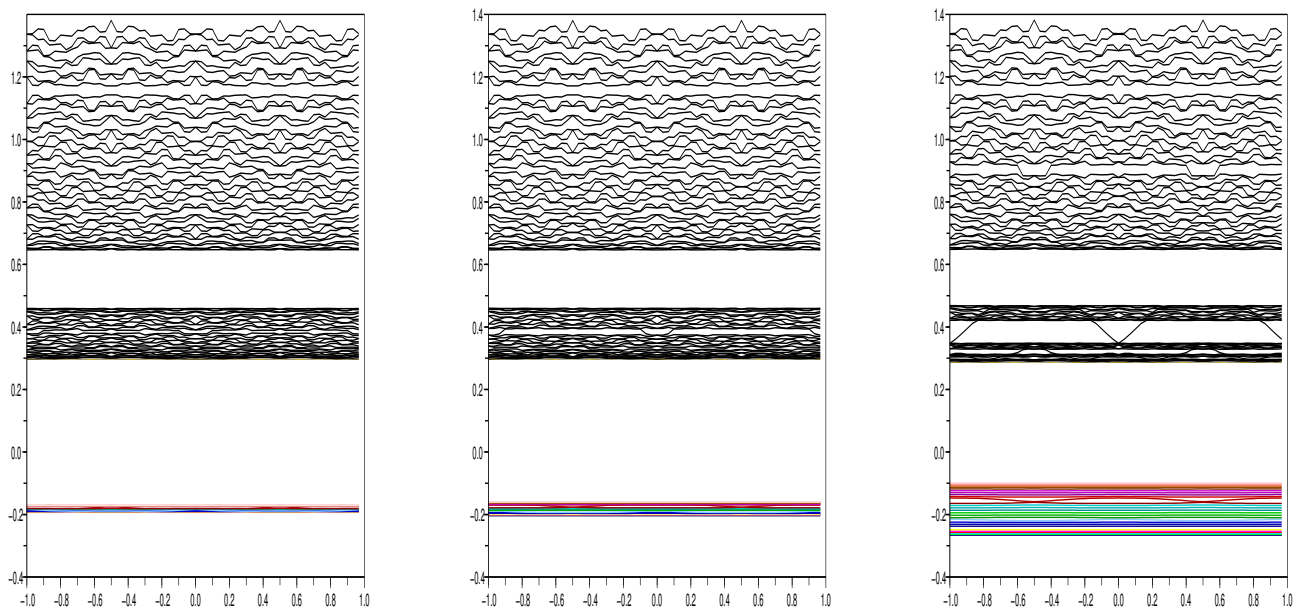

Fig. 4. Spectrum with $k=\frac{-1+\sqrt{5}}{2}$ and $\varepsilon=0.02$ (left), $\varepsilon=0.05$ (middle), $\varepsilon=0.2$ (right).

However, we ran the algorithm (16) with another value of $k=\frac{1}{2}(-1+\sqrt{5})$ in order to check its behavior in case the quasi-periodic perturbation is endowed with a frequency lower than the one of the underlying periodic potential. The numerical outcome is shown on Fig. 4; one notices at once the striking difference compared to Figs. 2 and 3, namely the absence of any "new band". This suggests that the former results may not be spurious as (16) is able to produce neat band gaps in the quasi-periodic regime (31 Fourier modes have been used in this computation). One can also consult $[44,20]$ where seemingly parabolic bands are being observed in a similar context.

An heuristic explanation can be given relying on [31] where it is argued that band gaps in the spectrum are associated to long-range order in the potential, i.e. usually periodicity, see e.g. [24] for a rigorous illustration of this fact. However, even if one cannot expect to obtain forbidden energies for a completely disordered system, it is known that for small enough perturbations, gaps still appear in the spectrum. More precisely, we recall Theorem 3.1 from [31]:

Theorem 3 Let $\mathcal{H}_{0}=-\frac{1}{2} \partial_{x x}+V(x)$ with $V$ periodic and $\mathcal{H}_{\delta}=-\frac{1}{2} \partial_{x x}+V(x+\delta h(x))$ with $h$ a $C^{3}(\mathbb{R})$ function having all its derivatives uniformly bounded by 1 . Suppose $[\alpha, \beta]$ belong to the resolvent set of $\mathcal{H}_{0}$, then for $\delta$ small enough, there exist $\alpha \leq \alpha_{\delta} \leq \beta_{\delta} \leq \beta$ such that the same property holds for $\left[\alpha_{\delta}, \beta_{\delta}\right]$ and $\mathcal{H}_{\delta}$. Moreover,

$$
\lim _{\delta \rightarrow 0} \alpha_{\delta}=\alpha, \quad \lim _{\delta \rightarrow 0} \beta_{\delta}=\beta
$$


For $\delta>0$, periodicity is lost, but the result somewhat expresses the fact that a weaker short-range order still exists in $\mathcal{H}_{\delta}$. Of course, long-range order is recovered in the limit $\delta \rightarrow 0$. In the present quasi-periodic context, we can think about using Theorem 3 with a linearized potential $Q(\vec{\omega} x):=V(x)+\delta h(k x)$, for which the basic frequencies are $\vec{\omega}=(1, k)$ (this situation is related to the one considered in [13], §IV.2). For $k \leq 1$, the perturbation is sufficiently slowly-varying in order to maintain band gaps like in Fig. 4, but for $k>1$, the result no longer applies and the spectrum's structure becomes more complex. Assuming that the "small perturbation" in $\varepsilon \cos (k x)$ stands for the smoothered Coulomb interaction with impurities molten in the periodic lattice, one may be tempted to call the new bands impurity bands, following the terminology of [27]. A possible interpretation of this phenomenon showing up for $k \geq 1$ in this context is the overlapping of wave functions when more that one impurity appears in an elementary cell. These metastable states are of great use in real-life applications such as LASERs and fluorescent light emitters.

\section{Time-dependent model in the incommensurate case}

Owing to the preceding results, we won't try to explore the Cantor-like structure of the "collapsed bands"; since a Cantor set is infinite, but nowhere dense, it is impossible to get it computed accurately in practice (one may just say that something messy appears here or there inside the spectrum); we recall that a Cantor spectrum obtained from (8) has positive measure. Moreover, it isn't possible to label the bands in the absolute; only can be done to label the "new bands" among themselves.

\subsection{2-D periodicity: reciprocal lattice and Bloch ansatz}

We are now concerned with the study of the band structure for (1) with the linearized potential (2). Since it is endowed with some two-dimensional periodicity properties (3), it becomes completely possible to follow the canvas of the standard Bloch decomposition in order to handle the problem, whatever the values of $k \in \mathbb{R}-\mathbb{Q}$ and $\varepsilon>0$. Let us define the periodic lattice for $V(t, x)$ in $\mathbb{R}^{2}$ as spanned by the two vectors:

$$
\vec{a}_{1}=(2 \pi k / \Omega(k), 2 \pi)^{T}, \quad \vec{a}_{2}=(2 \pi / \Omega(k), 0)^{T}
$$

The reciprocal lattice is spanned by $\vec{b}_{1}$ and $\vec{b}_{2}$ such that $\left.<\vec{a}_{i}, \vec{b}_{j}\right\rangle=2 \pi . \delta_{i, j}$, the Christoffel symbol. This yields:

$$
\vec{b}_{1}=(0,1)^{T}, \quad \vec{b}_{2}=(\Omega(k),-k)^{T} .
$$

These vectors are useful to write down the Fourier series for the potential; indeed,

$$
V(t, x)=\sum_{m, n \in \mathbb{Z}^{2}} \hat{V}_{m, n} \exp (i m x) \exp (i n(k x-\Omega(k) t) x)
$$

with

$$
\hat{V}_{ \pm 1,0}=\frac{1}{2}, \quad \hat{V}_{1, \pm 1}=\mp \frac{\varepsilon}{4}, \quad \hat{V}_{-1, \pm 1}= \pm \frac{\varepsilon}{4} .
$$

This produces a 2-D Brillouin zone (see [43] for some illustrations). However, we observe that there's no second derivative in time inside (1), thus the eigenvalue problem repeats itself exactly when $t \rightarrow t+2 \pi / \Omega(k)$. Thus there is no quasi-periodic boundary condition in the time variable, and the Bloch ansatz reads simply

$$
\Psi(\kappa, x)=\exp (i \kappa x) z_{\kappa}(t, x),
$$


the modulation $z_{\kappa}$ having the same periodicity as $V$, hence it can be written as a similar Fourier series. There is only one index $\kappa$ which belongs to the interval

$$
\kappa \in\left[-\frac{\max (1,|k|)}{2}, \frac{\max (1,|k|)}{2}\right] .
$$

Following the last step in [15], we obtain an algorithm which is very close to the twodimensional version of (11): (we dropped the $\kappa$ index for clarity here)

$$
\begin{gathered}
\left((m+n k+\kappa)^{2}-2 E\right) \hat{z}_{m, n}+\hat{z}_{m-1, n}+\hat{z}_{m+1, n}+ \\
\frac{\varepsilon}{2}\left(\hat{z}_{m-1, n+1}-\hat{z}_{m-1, n-1}+\hat{z}_{m+1, n-1}-\hat{z}_{m+1, n+1}\right)=0 .
\end{gathered}
$$

Once again, it is possible to obtain a structured matrix on which iterative diagonalization routines can reveal their efficiency. It is because of the similarity between (11), (16) and (18) that we wrote at the beginning of $\S 2$ that both quasi-periodic and 2-D periodic approaches lead to the same algorithm. Remark however that presently, Diophantine assumptions on $k$ shouldn't be needed in order to ensure the existence of Bloch wave solutions.

\subsection{The corresponding band structure: crossing levels}
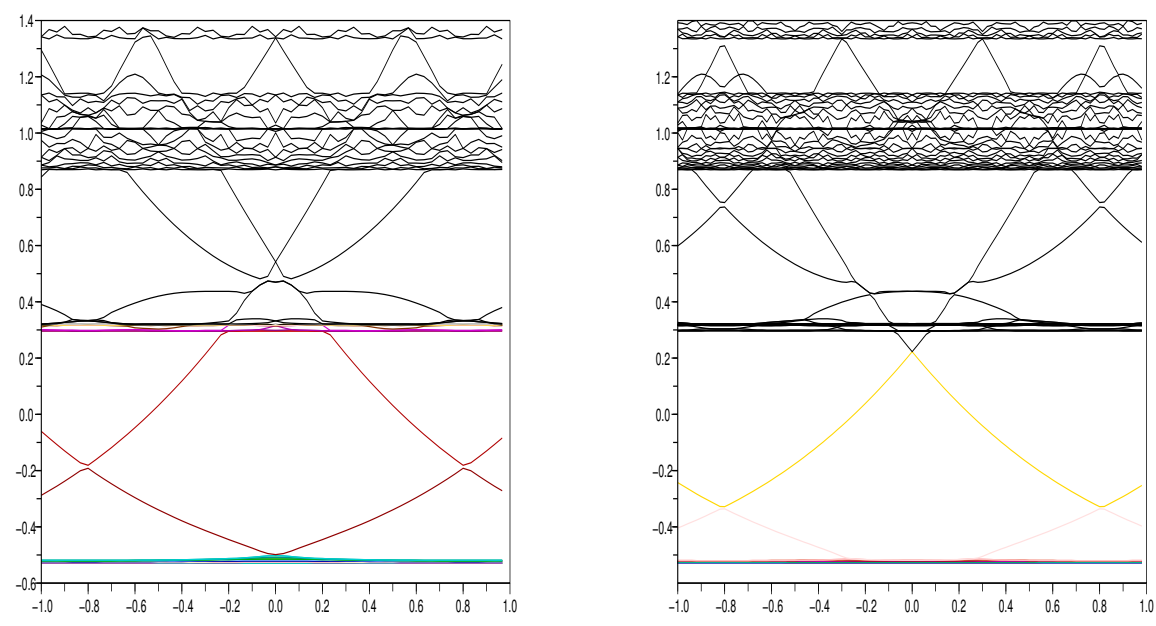

Fig. 5. Band structures with $n, m \in\{-15,15\}$ (left) and $n, m \in\{-25,25\}$ (right).

On Fig. 5, we display the band structure obtained out of (18) for both $n, m \in\{-15,15\}$ (left) and $n, m \in\{-25,25\}$ (right; this one asked for several CPU-days to get done) with the value $k=\frac{2}{\sqrt{5}-1}$. This shows that involving many Fourier modes in the computation doesn't lead to qualitative changes in the solution, especially the "Cantor zones" stay in the same places. The nature of the "new bands" remain also roughly the same; the main difference is that the ones being closest to a "Cantor zones" seem to flatten.

On Fig. 6, we present a zoom on the "new bands", especially in the vicinity of their crossing points. The lower ones cross only at the extremities of the interval $\left[-\frac{k}{2}, \frac{k}{2}\right]$ or in $k=0$, but the higher ones cross elsewhere. Now, this last feature might be a consequence of a shortage of Fourier modes: when passing from $n, m \in\{-15,15\}$ to $n, m \in\{-25,25\}$, some of the "new bands" seem to flatten (this is visible on the bottom of Fig. 6, somewhat less on the 

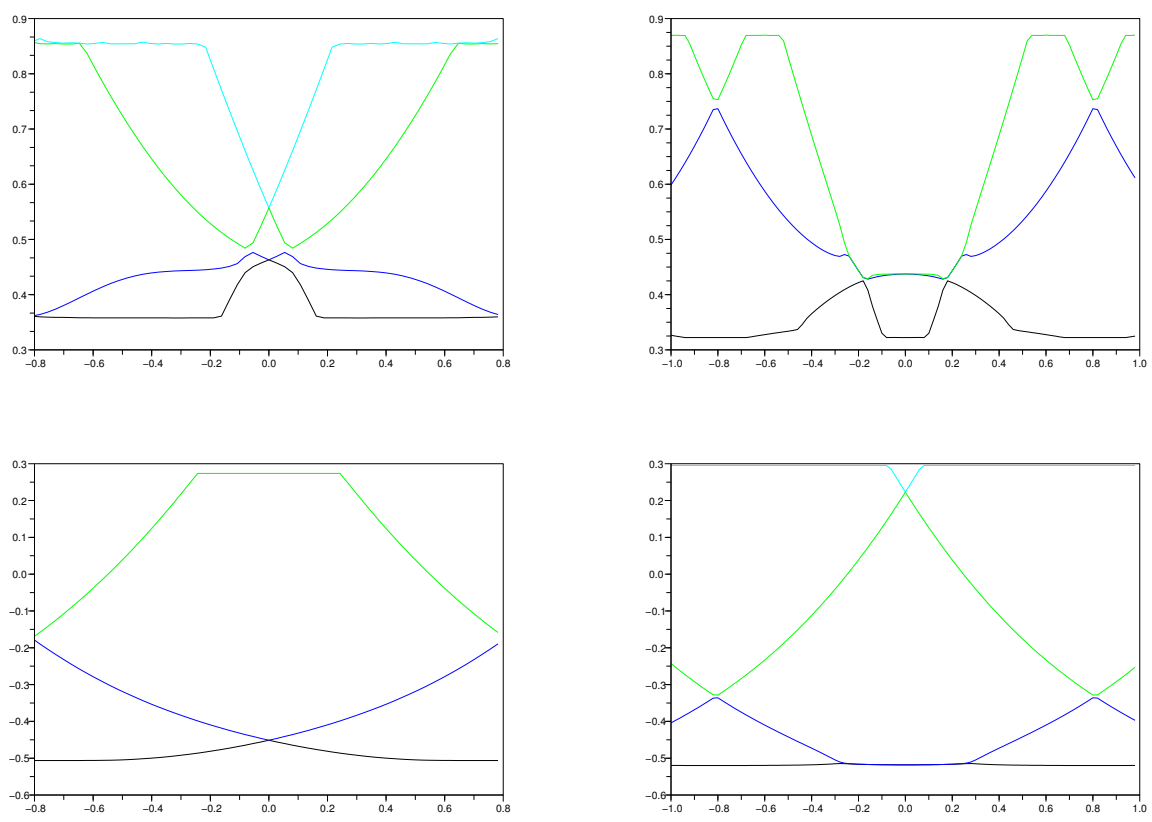

Fig. 6. Band crossings with $n, m \in\{-15,15\}$ (left) and $n, m \in\{-25,25\}$ (right).

top). It might therefore be that, for a even higher set of Fourier modes, only remain pieces of the $k$-periodic parabolas displayed on the left of Fig. 8 separated by Cantor-like zones.
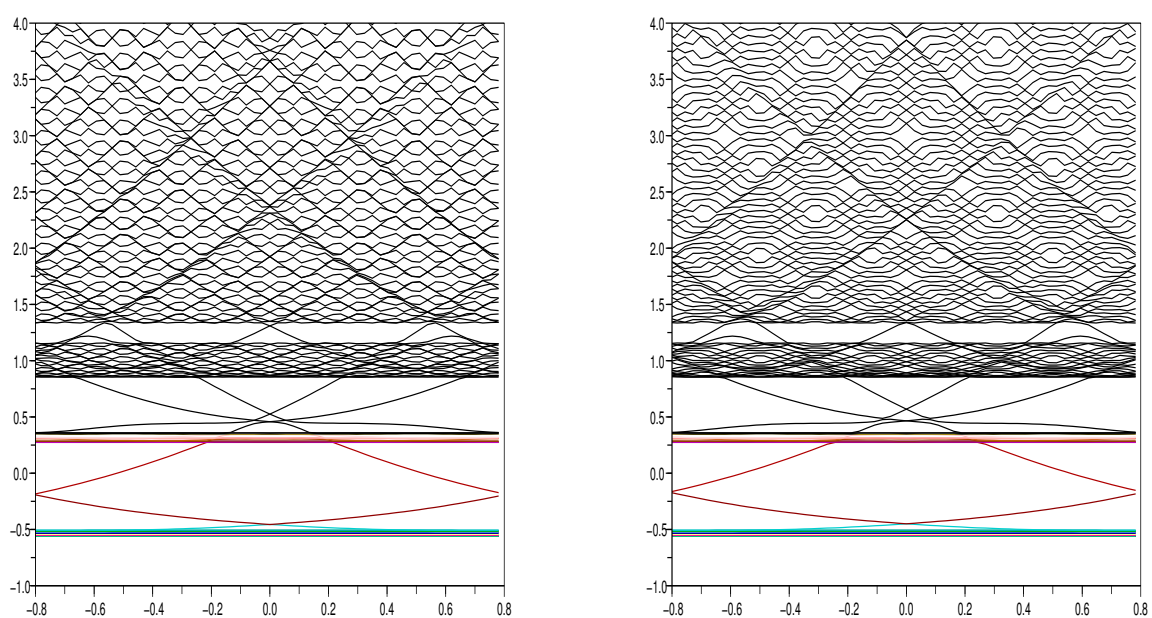

Fig. 7. Upper spectrum for $n, m \in\{-15,15\}: k=21 / 13$ (left) and $k=34 / 21$ (right).

Even if we don't want to explore what lies inside the "Cantor zones", we display on Fig. 7 the band structures for two Fibonacci approximants of the golden mean $k=\frac{2}{\sqrt{5}-1}$ computed by means of (18) with $n, m \in\{-15,15\}$. One observes that the upper part of the spectrum respects a periodicity in $\kappa$, even if it becomes more and more intricate. The amazing thing is that a parabolic structure seems to emerge in this area (as also suggested in [6], Fig. 6). 
It has been suggested in [6] the possibility that an electron represented by a wave-packet whose motion is described by (1) may nearly not feel the quasi-periodic potential and then display a quadratic dispersion relation. The mere influence of the vibrating lattice would be noticeable only through an effective mass which would significantly differ from the one calculated out of the conduction band of the periodic lattice at rest. We follow this idea here and recall that the value $m^{*} \simeq 0.28$ has been obtained for the static case in [15]. In Fig. 8, we juxtapose a parabolic approximation with the band picture where all the "Cantor states" have been removed. The agreement is quite interesting, but the values of the effective masses are notingly higher: we have $m^{*} \simeq 1.8$ for the lowest states and $m^{*} \simeq 0.98$ for the higher ones.
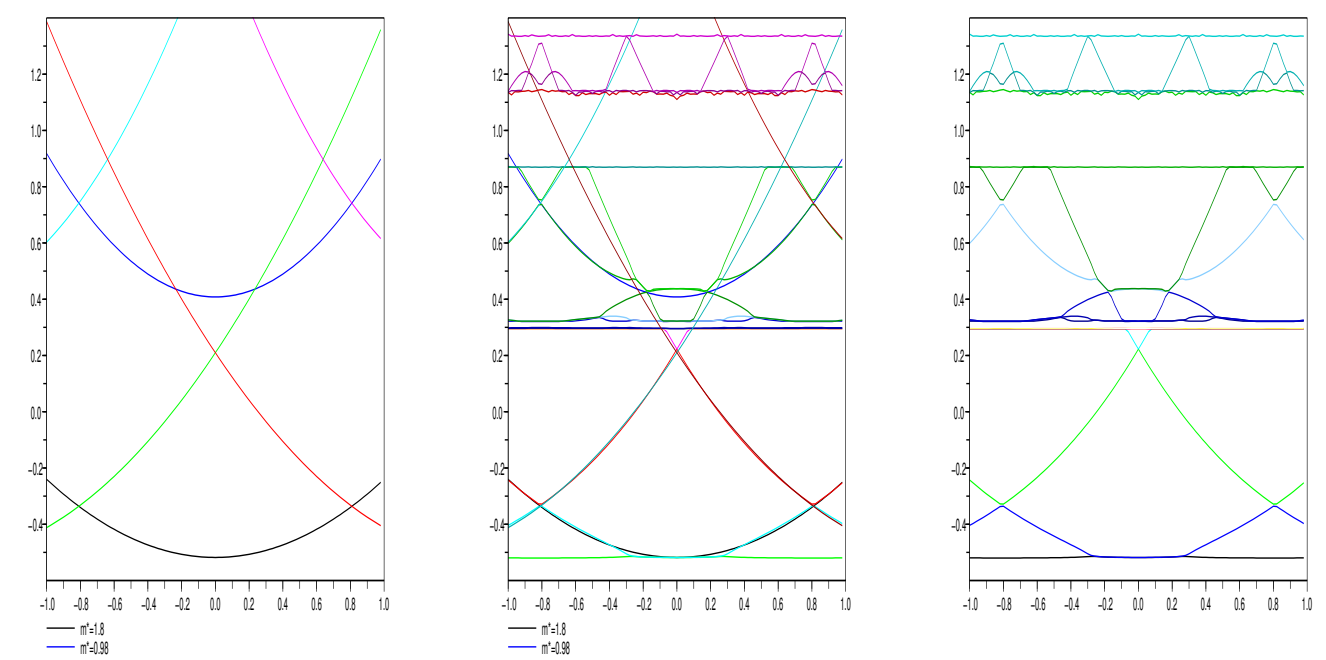

Fig. 8. Band structure for the incommensurate model: parabolic approximations periodically repeated (left), real values coming from the spectral algorithm (right) and superimposed (middle).

The parabolic dispersion framework is appealing in terms of computational costs: indeed, it has already been explained in [15] that this allows for a direct implementation of shockcapturing routines to simulate the time-evolution of WKB wave-functions. However, the obstruction on this route can be formulated as follows: which modulation should be used to form the ansatz in case a band-crossing occurs ? (see e.g. [10])

\subsection{Associated modulations: critical and extended states}

In this section, we are about to recall some of the features observed in [23,26], that is to say, localized, critical and extended eigenstates. A state is said to be localized if it belongs to $L^{2}(\mathbb{R})$ and its position density decays exponentially when $|x| \rightarrow+\infty$. On the contrary, it is extended when its $L^{2}(\mathbb{R})$ norm is infinite. Critical states refer to those quasi-Bloch waves which position density exhibits spikes arranged in a periodic manner in the spacetime plane. Our algorithm (18) is unable to compute localized states, as it is meant to look for periodic modulations $z_{\kappa}(t, x)$ associated to some eigenvalue $E_{\kappa}$. However, we can see on the top of Figs. 9 and 10 that critical states do show up; namely they are the ones associated with the two lowest "Cantor zones" in the band structure. 

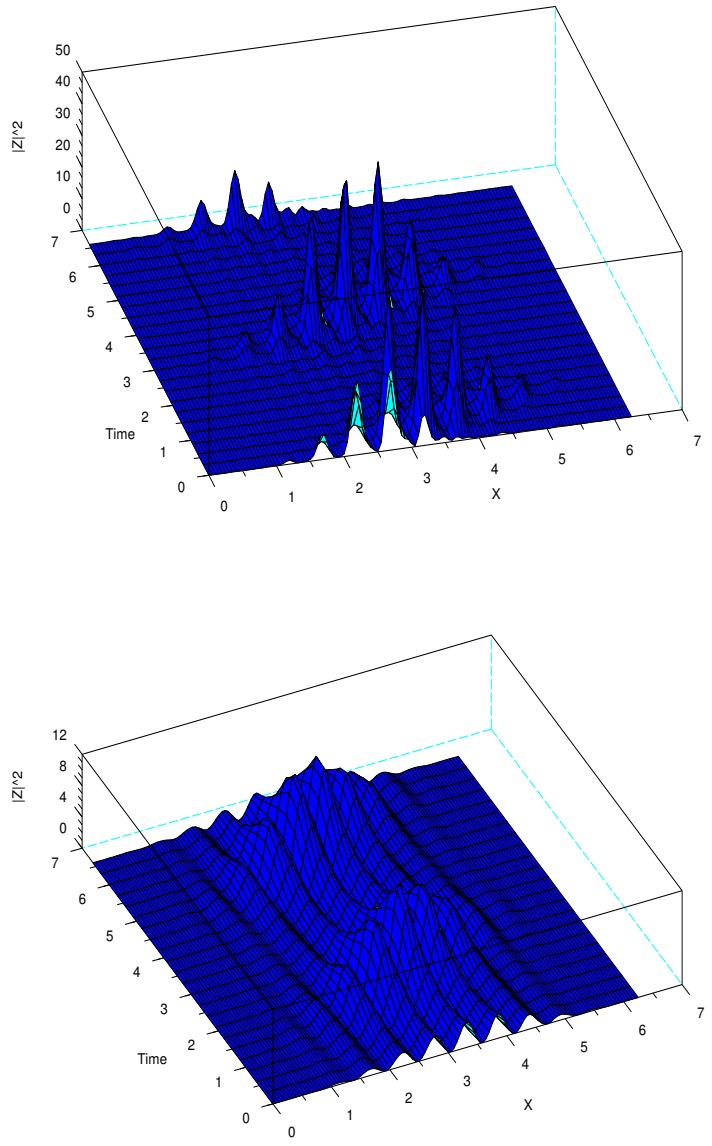

Fig. 9. Position density for increasingly energetic modulations with $\kappa=0$ : ground state (top) and extended one corresponding to the lower parabola (bottom).

In sharp contrast, the eigenstates associated to the "new parabolic bands" are extended, as can be seen on the bottom of Figs. 9 and 10. Moreover, one may observe that their position densities behave like the simpler ones in [15], Fig. 2: more precisely, the extended states corresponding to lower energies tend to be supported inside the potential well (even if it moves in time) whereas the higher ones are located above. The same seems to hold also for critical states, see Fig. 10 which displays spikes located on the potential's banks.

We found interesting to observe that critical eigenstates can actually be found at energy levels higher than extended ones. Indeed, to the critical state on top of Fig. 10 corresponds a "Cantor band" higher in the structure than the parabola associated to the extended state in the bottom of Fig. 9. This hasn't been noticed in [26].

On Fig. 11, we display the iso-lines of position densities associated to certain eigenstates with $\varepsilon=0.15$ : the left one refers to the lowest extended state, which is mainly situated in the vincinity of the potential's minima. The middle one is related to the highest critical state in the second "Cantor zone": one can notice how it follows the potential's high iso-lines. The right one corresponds to the extended state located immediately above in the spectrum; it is mainly located outside the potential's minima too, but its structure is obviously very different as it may be a good candidate to figure a conduction electron. They all correspond to the 3-D representations of Figs. 9 and 10. Higher eigenstates reveal more uniformly located oscillations. 

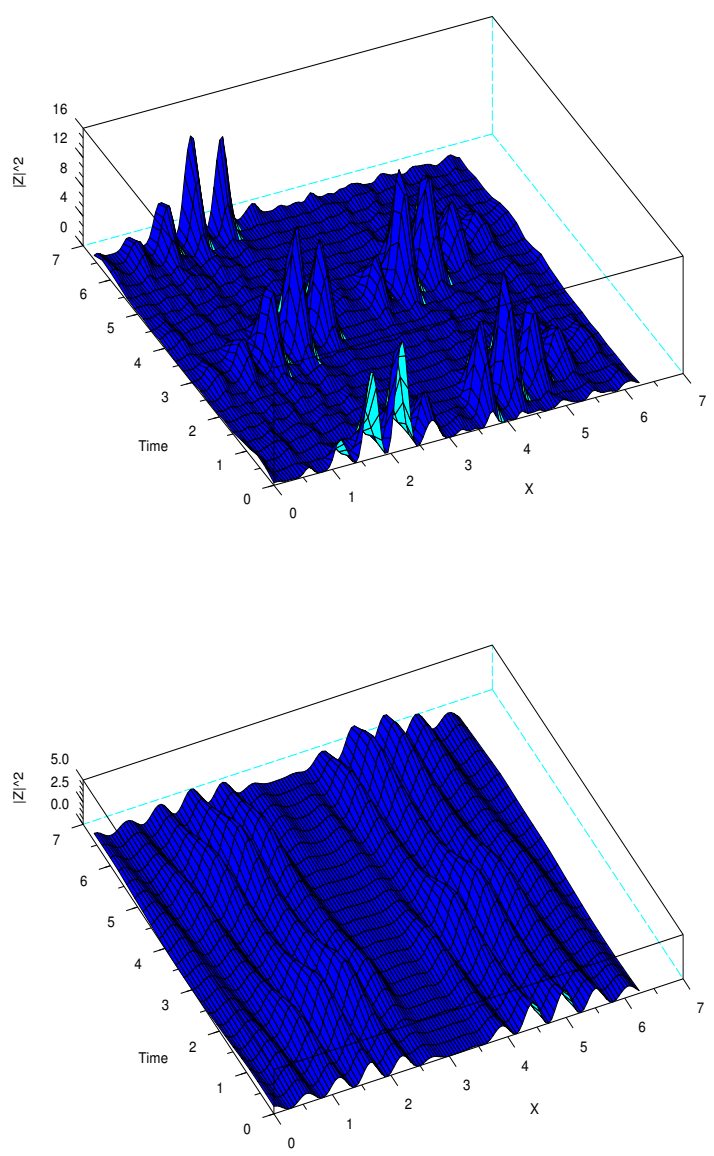

Fig. 10. Position density for increasingly energetic modulations with $\kappa=0$, continued: critical state in the intermediate Cantor set (top) and extended one corresponding to the higher parabola (bottom).
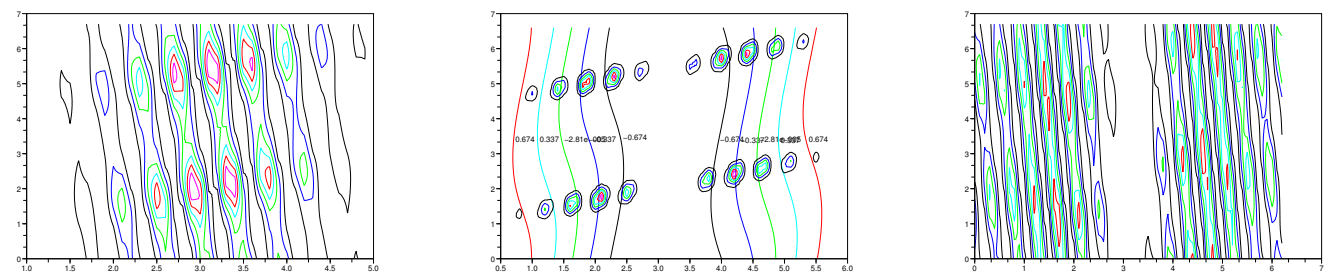

Fig. 11. Lower extended (left), critical (middle) and higher extended (right) eigenstates. (absissae are for space, ordinates for time)

We didn't observe any localized states like in [26]; however, their presence in the spectrum could be questionable as the potential (2) is genuinely 2-D periodic. Hence its eigenstates should be given by 2-D Bloch waves having only the particularity of being exactly periodic in time. However, the position densities of the critical states we found by means of (18) follow nicely the iso-lines of the potential (2). We shall see in the next section that this is quite a robust property since it still holds while changing the value of the parameter $\varepsilon$ which controls the strength of the modulation: a stronger modulation giving a more oscillating 
potential.

4.5 Effects of the modulation's strength: from $\varepsilon=0.05$ to $\varepsilon=0.5$
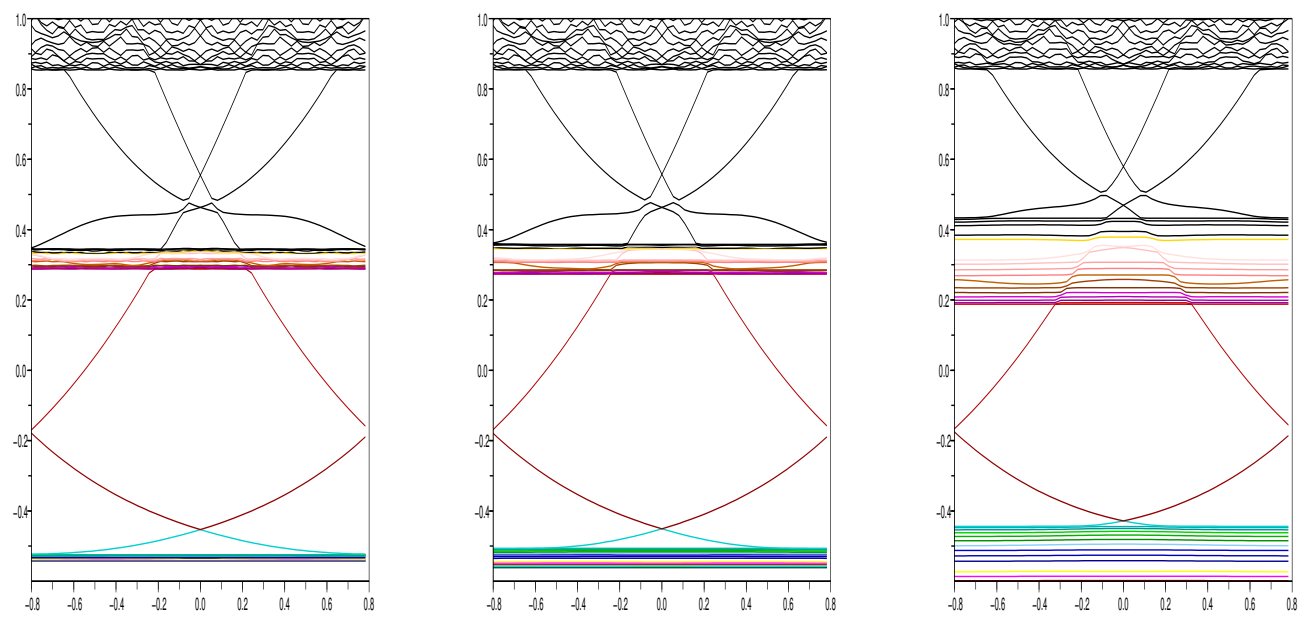

Fig. 12. Band structures for $\varepsilon=0.05$ (left), $\varepsilon=0.15$ (middle) and $\varepsilon=0.5$ (right).

Increasing the value of the parameter $\varepsilon$ doesn't seem to change the structure of the overall band picture: namely, it widens the "Cantor-like zones" inside the spectrum, as can be seen on Fig. 12 with $n, m \in\{-15,15\}$. The "new bands" aren't much changed during the process, except that their interval of variation has shrinked. One can imagine that for a really big value of $\varepsilon$, no band can show up no more, and the spectrum will therefore display a completely singular structure, like on the top of Fig. 7 (or in [26], Fig. 1). The associated eigenfunctions would display the Anderson localization.
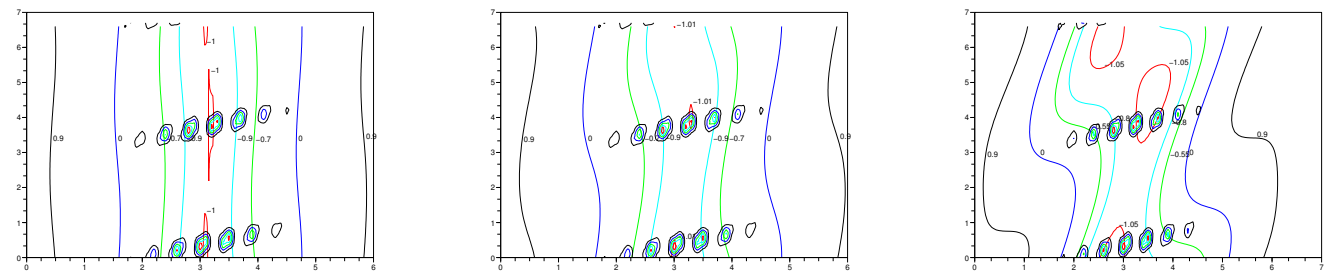

Fig. 13. Ground states superimposed with potential's iso-values for $\varepsilon=0.05$ (left), $\varepsilon=0.15$ (middle) and $\varepsilon=0.5$ (right).

For completeness, we also displayed the behavior of the (critical) ground state when $\varepsilon$ is increased on Fig. 13. The main observation is that the spikes keep on being located on the iso-values of the potential, even when it shows quite severe oscillations in the space/time plane. This suggests that the whole algorithm (18) produces solutions which remain consistent with the data, even if they reveal a more singular structure than e.g. the Bloch waves in [14]. 


\section{$5 \quad$ What happens for different values of $k$ ?}

We have seen in the last two sections that for both static and time-dependent models, "new bands" appear and look like being $k$-periodic. Hence it might be that reducing the value of $k$ is likely to make them more difficult to observe, like in $\S 3.3$ (however, the interpretation as impurity bands doesn't really hold here). This is what we want to study in this section working with the inverse $k=\frac{\sqrt{5}-1}{2}$. We shall also have a look at $k=\frac{\pi}{3}$, for which the "parabolic structure" previously discussed should appear.

\section{$5.1 k$ is smaller than one}

We still consider the potential (2) and we use (18) to investigate numerically its eigenvalues in case $k=\frac{\sqrt{5}-1}{2} \leq 1$ : the results are shown on Fig. 14. It is interesting to observe that now, band gaps are easily noticeable and for $\varepsilon=0.05$, they are located approximately like in the unperturbed case, see Fig. 1 in [15]. When $\varepsilon$ increases, more gaps of smaller size open in the spectrum; this is somehow similar to what we observed on Fig. 12. However, the big difference is that new bands don't seem to show up like before; this is again consistent with the results obtained with the static model and displayed on Fig. 4.
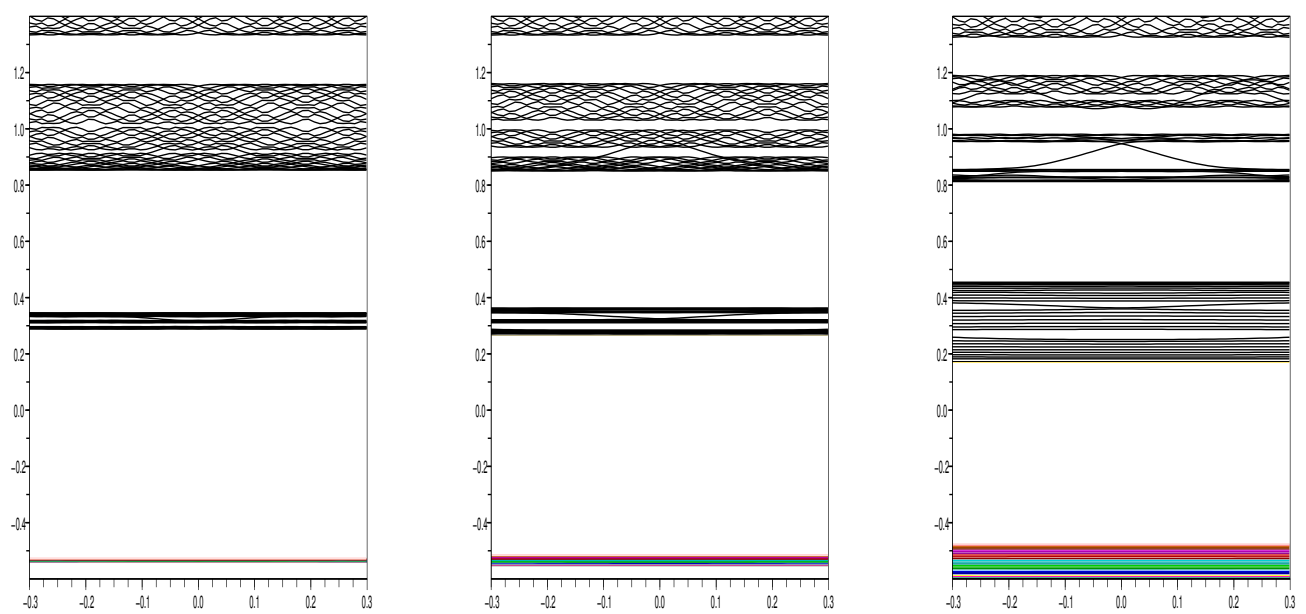

Fig. 14. Spectrum for $\varepsilon=0.05$ (left), $\varepsilon=0.15$ (middle) and $\varepsilon=0.5$ (right) with $k=\frac{\sqrt{5}-1}{2}$.

"New bands" might really be a quasi-periodic phenomenon which appearance stems only from the set of basic frequencies present in the potential $V$. In both cases given by (2) and (15), basic frequencies are $\vec{\omega}=(1, k)$ at any fixed time $t$; "new bands" could develop better with $|k|$ getting bigger, meaning that the (small) perturbation term oscillates at a frequency higher than the one of the lattice at rest. This gives for both potentials a threshold value of $\bar{k} \simeq 1$, in agreement with the numerical results. On the contrary, owing again to Figs. 4 and 14 , the number of band gaps seems to increase when $\varepsilon /|k|$ grows.

We also present position densities for some eigenstates chosen around some energy level like in Fig. 11; results are shown in Fig. 15. One sees that as $\varepsilon$ grows, these states pass from being "nearly extended" (for $\varepsilon=0.05$ ) to clearly critical (for $\varepsilon \geq 0.15$ ): this may indicate 
a locally smooth dependence in $\varepsilon$. The energy level we considered here corresponds to the eigenstate displayed on the right of Fig. 11.
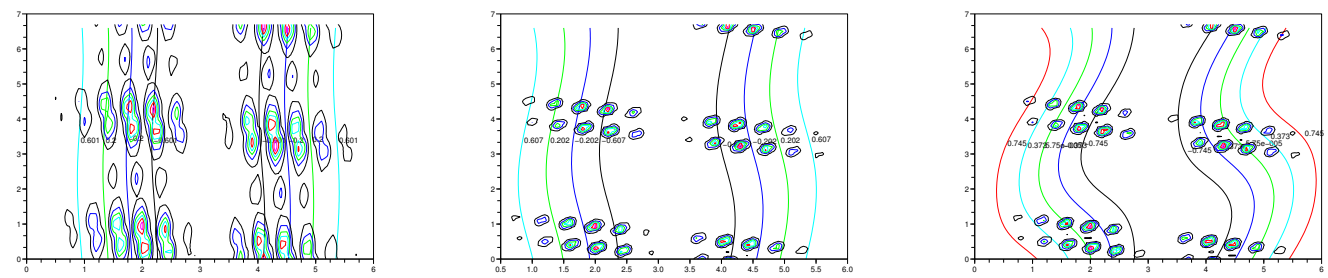

Fig. 15. Eigenstates with $k=\frac{\sqrt{5}-1}{2}$ for $\varepsilon=0.05$ (left), $\varepsilon=0.15$ (middle) and $\varepsilon=0.5$ (right).

\section{2 $k$ is bigger than one}

In order to decide whether the (incommensurate) value of $k$ has an influence on the "new bands", we ran the algorithm (18) with $k=\frac{\pi}{3} \geq 1$ and $\varepsilon=0.15$. Looking at Fig. 16, one can see the big difference compared to Fig. 14: no band gaps remain, and the upper part of the spectrum doesn't really appear like a Cantor set anymore. Seemingly "parabolic new bands" are visible and display a $k$-period as in the previous sections; there is some sort of similarity with Fig. 7. (41 Fourier modes have been used in this computation)
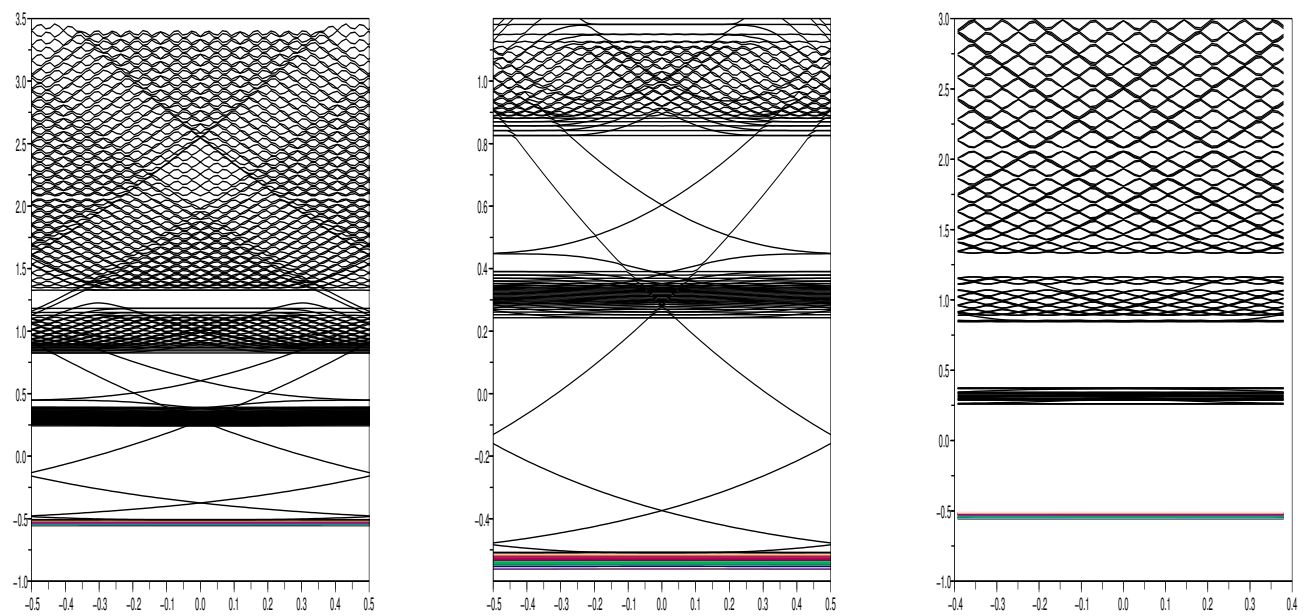

Fig. 16. Spectrum for $k=\frac{\pi}{3}, \varepsilon=0.15$ : full picture (left) and detail (middle); comparison with $k=\frac{\pi}{4}$ (right).

\section{Conclusion}

In order to compute accurately the spectrum of a Schrödinger equation modelling a onedimensional modulated (or deformed) incommensurate crystal without any tight-binding approximation, we derived in this paper a spectral algorithm which can be built out of the 
recent theory of quasi-periodic functions or by taking advantage of periodicity properties of the potential in a $2-\mathrm{D}$ space. The numerical results revealed several unexpected features:

- New "seemingly parabolic bands" emerge in the spectrum. The band diagram is therefore split into "Cantor-like" domains which result from the band nesting phenomenon, [21] and these "impurity bands" which turn out to be $k$-periodic. When computing the corresponding effective masses, one finds values much bigger than the ones coming from the usual conduction band of the unperturbed lattice (in our case, the Mathieu equation treated in [15-17]). Hence two competing effects appear: metallic conductivity can be achieved this way, like e.g. in highly-doped semiconductors, but scattering effects due to quasi-periodicity are important.

- Two different types of eigenstates appear: in the terminology of [23,26], we speak about critical states generally found in the bottom of the spectrum and always associated with a Cantor-like area. These states are very peaked and their spikes are located on the isolines of the potential. The other type of eigenstate is the more usual extended state, which looks essentially like a Bloch wave: those are the only ones which may represent conduction electrons.

- The value of the wave number $k$ in $(2)$ is very important in many aspects: even if one assumes it is Diophantine, its magnitude has a huge influence on the overall spectrum picture, recall e.g. the Figs 14 and 16: the threshold value in this case looks like being $k \simeq 1$, which means that "new bands" show up only in case the frequency of the small perturbation is higher than the one of the underlying periodic lattice. This seems to be supported by Theorem 3.1 in [31], which holds in multi-dimension and states essentially that weak and slowly-varying deformations of periodic potentials still admit band gaps.

This suggests that a semiclassical WKB approximation to (1) should take into account for these features. The main obstruction in this direction lies in understanding what happens at the band-crossing points, since it looks clear from Fig. 7 and 16 that the parabolas extend very far inside the band diagram on the top of the spectrum.

\section{A Numerical visualization of a continuous "almost-Mathieu" model}

For completeness, and even if this case doesn't exactly fit in our general framework ${ }^{7}$, we display here the numerical outcome of the algorithm written in [40] to solve the problem (13); it is just a special case of (11). Namely what we want to check is the ability of this numerical processing to recover well-known results, like e.g. the existence of band gaps for $\varepsilon$ small enough. Unfortunately, it isn't possible to achieve conclusive computations for $\varepsilon \leq 0.1$ with a reasonable number of Fourier modes (typically, between 31 and 51) because the corresponding outcome is blurred with spurious oscillations (like on the left of Fig. A.1). In this figure, one sees that for $\varepsilon=0.5$, a lot of eigen-states are constant in $\kappa$ but, for all values of $\varepsilon$, at least one band gap shows up in the structure. For $\varepsilon>0.7$, the algorithm becomes unstable; we recall that in this case, the parameter $\varepsilon$ acts on the whole potential, which isn't really a perturbated periodic function no more.

We finally wanted to observe what happens for a small value of $k$ in this case, and we took again $k=\frac{\sqrt{5}-1}{2}$; results are displayed in Fig. A.2. This seems to have no effect on the presence of "new bands", whose presence can be questionable as the lower ones on Figs. A.1 and A.2 appear inside a range of energies where the computations reported in $[4,46]$ predict a band gap. They don't seem to be neither parabolic nor $k$-periodic as in the former sections, hence we think they are spurious. Actually, our outcome with $\varepsilon=0.5$ could also be compared with Fig. 3 in [6] where periodic approximations are studied by means of a spectral scheme like in [15]; the corresponding potential is displayed on Fig. 2 in the same

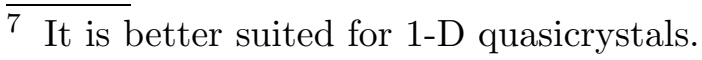



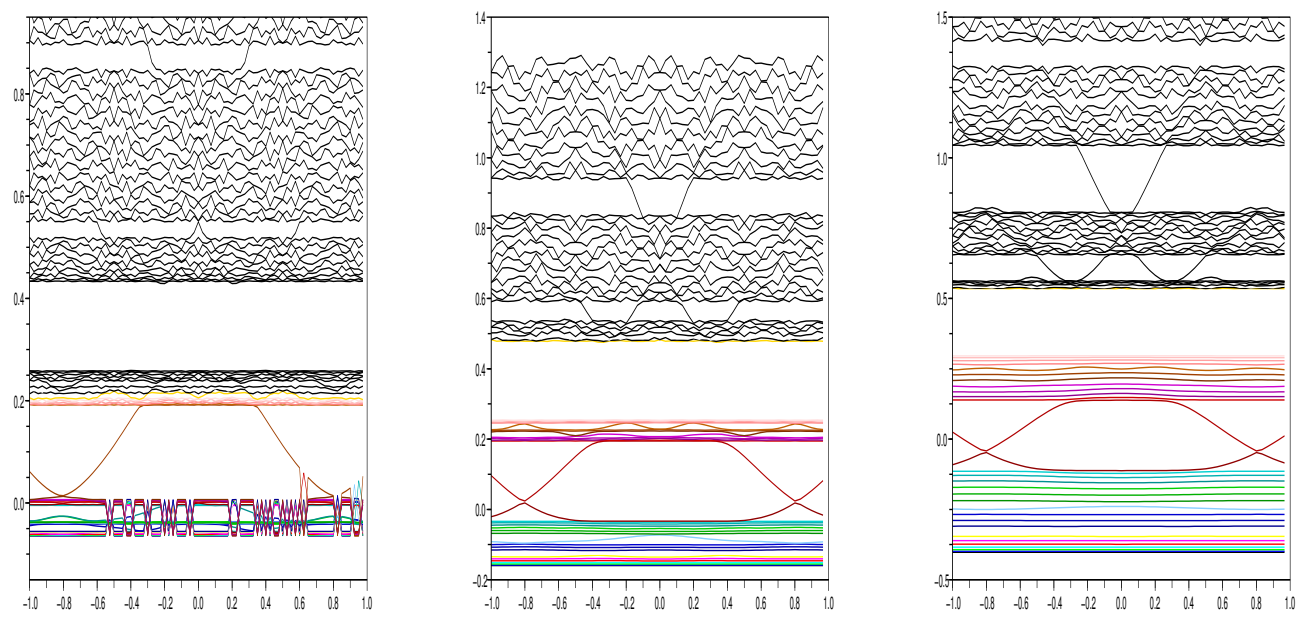

Fig. A.1. Spectrum for Surace's model with $k=\frac{2}{\sqrt{5}-1}$ : inconclusive $\varepsilon=0.1$ (left), $\varepsilon=0.3$ (middle) and $\varepsilon=0.5$ (right).

article. Similarly, something appears in the band gaps for another case study of the type (13) reported in [21]: see Fig. $3, p / q \simeq 30 / 61$ and $p / q \simeq 31 / 61$
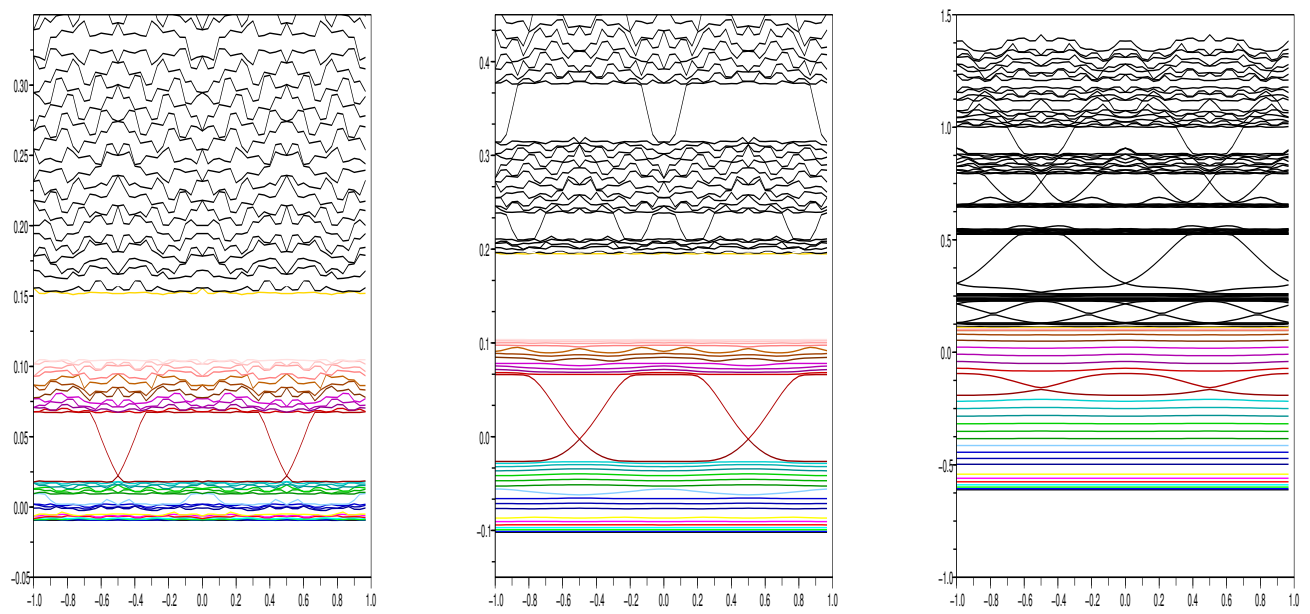

Fig. A.2. Spectrum for Surace's model with $k=\frac{\sqrt{5}-1}{2}: \varepsilon=0.05$ (left), $\varepsilon=0.15$ (middle) and $\varepsilon=0.5$ (right).

In order to provide some explanation about the figures A.1 and A.2, we turn back to the notion of "resonance tongue", see Definition 2 and [3,4]. In case the eigenvalue problem is given by (13), we observe that $\vec{\omega}=(1, k) \in \mathbb{R}^{2}$ thus the set of resonances is given by

$$
\frac{1}{2}(i+j k), \quad(i, j) \in \mathbb{Z}^{2},
$$

hence is dense in $\mathbb{R}$. Owing to the continuity of the rotation number $\beta$, we just want to check that the gaps we found in the preceding figures for $\varepsilon$ small do correspond to resonances of 
the form (A.1) for small values of $i, j$. Indeed on Fig. A.1, for both $\varepsilon=0.1$ and $\varepsilon=0.3$, we observe a gap roughly in $[0.25,0.45]$; this may emanate from the elementary resonance:

$$
\alpha=\frac{3}{2}(-1+k) \simeq 0.927051, \quad \frac{\alpha^{2}}{2} \simeq 0.4297118, \quad k=\frac{2}{\sqrt{5}-1} .
$$

As the frequency vector $\vec{\omega}$ is identical, we may think that the little gap around $E=0.4$ on both figures 2 and 3 has the same origin. A simpler resonance is proposed in Fig. 2 of [4] (according to their notation, $E=\frac{a^{2}}{2}$ and $\varepsilon=\frac{b}{2}$ ) for (13): it is based on the choice $i=0$, $j=1$ in (A.1) and yields $E \simeq 0.33$. It marches our results quite well (and also Fig. 5.1 in [46]) but one may prefer our former value because it meets also the outcome of $\S 3.2$. Concerning Fig. A.2, there seems to be a gap inside the interval $[0.1,0.15]$ for both $\varepsilon=0.05$ and $\varepsilon=0.15$; this may correspond to the most elementary resonance:

$$
\alpha=\frac{1}{2}(1+0 k)=0.5, \quad \frac{\alpha^{2}}{2}=0.125, \quad k=\frac{\sqrt{5}-1}{2} .
$$

Still, the basic frequencies vector $\vec{\omega}$ is the same compared to the one which led to the results of Fig. 4 even if the potential is different; nevertheless, we observe that a gap opens around the same energy value in that case. Looking at Figs. 2.1, 3.1, 5.1 and 5.2 in [46] while according to their notation $(\varepsilon=\varepsilon / 2$ and $E=\delta / 2)$, we see that very different algorithms led to the finding of a very stable gap (in terms of $k$ ) for this range of energies. Of course, this is too weak as a validation for our results. However, the fact that our gaps are stable for small values of $\varepsilon$ and are roughly located around commonly found resonances is rather encouraging.

Remark 3 The results in this appendix are related to the ones in [4] (and also in [46]); reference [4] concentrates on the eigen-problem (5) where $q(x)=\cos \left(\omega_{1} x\right)+\cos \left(\omega_{2} x\right)$ with incompatible frequencies. Different notations are used as their $\left(a^{2}, b\right)$ correspond here to $(E, \epsilon)$. Figures 1 and 2 in [4] are a different view of the band structure disregarding the $\kappa$-dependence of $E(\kappa, \epsilon)$ but emphasizing the influence of the size of $\epsilon$. Summarizing, in the present paper, we plot $\kappa \mapsto E$ for different values of $\epsilon$ (see Figs. A.1 and A.2) whereas $\epsilon \mapsto E$ is shown in Figs. 1 and 2 of [4]. Later, a study of instability pockets is conducted for $q(x)=\cos \left(\omega_{1} x\right)+\cos \left(\omega_{2} x\right)+\cos \left(\left(\omega_{1}+\omega_{2}\right) x\right)$, which isn't touched here. Similarly as [46] Figure 3.1, the authors of [4] study numerically the Lyapunov exponent $\lambda$ and the rotation number $\rho$ associated to (5), both as functions of $E$ in Figs. 4-7. At last, the remaining of [4] is concerned with a very detailed numerical study of phenomena near the collapse line of resonances, thus goes beyond the scope of the present work.

\section{References}

[1] N.W. Ashcroft and N.D. Mermin, Solid-state physics, Holt; Rinehart and Winston (1976).

[2] G. Benfatto, G. Gentile, V. Mastropiero, Electrons in a lattice with an incommensurate potential, J. Stat. Phys. 89 (1997) 655-708.

[3] H. Broer, J. Puig, C. Simó, Resonances tongues and instability pockets in the quasi-periodic Hill-Schrödinger equation, Comm. Math. Phys. 241 (2003) 467503.

[4] H. Broer, C. Simó, Hill's equation with quasi-periodic forcing: resonance tongues, instability pockets and global phenomena, Bol. Soc. Braz. Mat. 29 (1998) 253-293. 
[5] R. Carles, P. Markowich, C. Sparber, Semiclassical asymptotics for weakly nonlinear Bloch waves, J. Stat. Phys. 117 (2004) 343-375.

[6] R.B. Diener, G.A. Georgakis, J. Zhong, M. Raizen, Q. Niu, Transition between extended and localized states in a one-dimensional incommensurate optical lattice Phys. Rev. A 64 (2001) 033416.

[7] E. Dinaburg and Yakov Sinaï, The one-dimensional Schrödinger equation with quasi-periodic potential Funkt. Anal. i Priloz. 9 (1975) 8-21.

[8] L.H. Eliasson, Floquet solutions for the 1-dimensional quasi-periodic Schrödinger equation, Comm. Math. Phys. 146 (1992) 447-482.

[9] V.J. Emery, J.D. Axe, One-dimensional fluctuations and chain ordering transformations in $H_{3}{ }_{3} A s F_{6}$, Phys. Rev. Lett. 40 (1978) 1507.

[10] C. Fermanian-Kammerer, C. Lasser, An algorithm for quantum propagation through electron level crossings, preprint.

[11] J. Fröhlich, J. Pöschel, P. Wittawer, Localization for a class of one-dimensional quasi-periodic Schrödinger operators, Comm. Math. Phys. 132 (1990) 5-25.

[12] G. Gentile, D.A. Cortez, J.C.A. Barata, Stability for the quasi-periodically perturbed Hill's equation, Comm. Math. Phys. 260 (2005) 403-443.

[13] C. Gérard, A. Martinez, J. Sjöstrand, A mathematical approach to the effective Hamiltonian in perturbed periodic problems, Comm. Math. Phys. 142 (1991) 217244.

[14] L. Gosse, Multiphase semiclassical approximation of the one-dimensional harmonic crystal. I. The periodic case, J. Phys. A Math. Gen. 39 (2006) 1050910521.

[15] L. Gosse, P.A. Markowich, Multiphase semiclassical approximation of an electron in a one-dimensional periodic lattice. I. Homogeneous problems, J. Comp. Phys. 197 (2004) 387-417.

[16] L. Gosse, Multiphase semiclassical approximation of an electron in a one-dimensional crystalline lattice - II. Impurities, confinement and Bloch oscillations., J. Comp. Phys. 201 (2004) 344-375.

[17] L. Gosse and N.J. Mauser, Multiphase semiclassical approximation of an electron in a one-dimensional crystalline lattice - III. From ab-initio models to WKB for Schrödinger-Poisson., J. Comp. Phys. 211 (2006) 326-346.

[18] L. Gosse and O. Runborg, Finite moment problems and applications to multiphase computations in geometric optics, Comm. Math. Sci. 3 (2005) 373392.

[19] J.C. Guillot, J. Ralston, E. Trubowitz, Semiclassical asymptotics in solid-state physics, Comm. Math. Phys. 116 (1988) 401-415.

[20] J. Hafner, M. Krajči, Electronic structure and stability of quasicrystals: quasiperiodic dispersion relations and pseudogaps Phys. Rev. Lett. 68 (1992) $2321-2324$. 
[21] J.M. Hollingworth, A. Vourdas, N. Backhouse, Wave propagation in onedimensional optical quasiperiodic systems Phys. Rev. E 64 (2001) 036611.

[22] R. Johnson, J. Moser, The rotation number for almost periodic potentials, Comm. Math. Phys. 84 (1982) 403-438.

[23] M. Kohmoto, B. Sutherland, C. Tang, Critical wave functions and Cantor-set spectrum of a one-dimensional quasicrystal model, Phys. Rev. B 35 (1987) 10201033.

[24] Evgeny Korotyaev, Estimates on periodic potentials in terms of gap lengths, Comm. Math. Phys. 197 (1998) 521-526.

[25] C. de Lange, T. Janssen, Incommensurability and recursivity: lattice dynamics of modulated crystals J. Phys. C: Solid State Phys. 14 (1981) 5269-5292.

[26] C. de Lange, T. Janssen, Electrons in incommensurate crystals: spectrum and localization Phys. Rev. B 28 (1983) 195-209.

[27] Melvin Lax and James C. Phillips , One-Dimensional Impurity Bands Phys. Rev. 110 (1958) 41-49.

[28] W. Magnus, S. Winkler, Hill's equation, John Wiley \& Sons; New York (1966).

[29] W. Man, M. Megens, P.J. Steinhart, P.M. Chaikin, Experimental measurement of the photonic properties of icosahedral quasicrystals, Nature 436 (18 august 2005) 993-997.

[30] J. Moser, J. Pöschel, An extension of a result by Dinaburg and Sinai on quasiperiodic potentials Comment. Math. Helvetici 59 (1984) 39-85.

[31] G. Nenciu, Dynamics of band electrons in electric and magnetic fields: rigorous justification of the effective Hamiltonians, Rev. Mod. Phys. 63 (1991) 91-127.

[32] Q. Niu, Quantum adiabatic particle transport Phys. Rev. B 34 (1986) 50935100 .

[33] Q. Niu, X.G. Zhao, G.A. Georgakis, M.G. Raizen, Atomic Landau-Zener tunneling and Wannier-Stark ladders in optical potentials Phys. Rev. Lett. 76 (1996) 4504-4507.

[34] R.E. Peierls, Quantum theory of solids, Clarendon Oxford (1955).

[35] E. Peik, M. Ben Dahan, I. Bouchoule, Y. Castin, C. Salomon, Bloch oscillations of atoms, adiabatic rapid passage and monokinetic atomic beams Phys. Rev. A 55 (1997) 2989.

[36] J. Puig, Reducibility of linear equations with quasi-periodic coefficients: A survey Preprint (2002).

[37] J. J. Rehr and W. Kohn, Impurity states between two bands, Phys. Rev. B 9 (1974) 1981-1983.

[38] E. Rotenberg, W. Theis, K. Horn, P. Gille, Quasicrystalline valence bands in decagonal Al-Ni-Co, Letters to Nature 406 (10 august 2000) 602-605. 
[39] G. Sundaram, Q. Niu, Wave-packet dynamics in slowly perturbed crystals: gradient corrections and Berry-phase effects, Phys. Rev. B 59 (1999) 14915-14925.

[40] S. Surace Jr., The Schrödinger equation with a quasi-periodic potential, Trans. Amer. Math. Soc. 320 (1990) 321-370.

[41] M.E. Taylor, Partial Differential Equations. I. Basic theory, Springer.

[42] M.A. Torres, J.P. Adrados, J.L. Aragon, P. Cobo, S. Tehuacanero, Quasiperiodic Bloch-like states in surface wave experiment Phys. Rev. Lett. 90 (2003) 114501.

[43] J.J.P. Veerman, M.M. Peixoto, A.C. Rocha, S. Sutherland, On Brillouin zones, Comm. Math. Phys. 212 (2000) 725-744.

[44] J. Voit, L. Perfetti, F. Zwick, H. Berger, G. Margaritondo, G. Grüner, H. Höchst, M. Grioni, Electronic structure of solids with competing periodic potentials, Science 290 (2000) 501-503.

[45] W.M. Zheng, Renormalization procedure for the quasiperiodic Schrödinger equation, J. Phys. A: Math. Gen. 19 (1986) L715-L720.

[46] R. S. Zounes, R.H. Rand, Transition curves for the quasi-periodic Mathieu equation, SIAM J. Appl. Math. 58 (1998) 1094-1115. 